\title{
Research on Stiffness of Multibackbone Continuum Robot Based on Screw Theory and Euler-Bernoulli Beam
}

\author{
Bin He ${ }^{D}$, Shoulin $\mathrm{Xu}$, and Zhipeng Wang \\ Department of Control Science and Engineering, Tongji University, Shanghai 201804, China \\ Correspondence should be addressed to Bin He; hebin@tongji.edu.cn
}

Received 8 November 2017; Revised 10 April 2018; Accepted 15 April 2018; Published 16 May 2018

Academic Editor: John D. Clayton

Copyright (C) 2018 Bin He et al. This is an open access article distributed under the Creative Commons Attribution License, which permits unrestricted use, distribution, and reproduction in any medium, provided the original work is properly cited.

\begin{abstract}
Continuum robots have become a focus for extensive research, since they can work well in complex and confined environments. The main contribution of this paper is to establish a stiffness model of a single section multibackbone continuum robot and analyze the effect of the structural parameters of continuum robot on the overall rotation and translation stiffness. First, a stiffness model which indicates the end configuration of continuum robot under external load is deduced by the screw theory and Euler-Bernoulli beam. Then, the stiffness elements are fully analyzed, therefore, obtaining the influence of the structural parameters of continuum robot on the stiffness elements. Meanwhile, a numerical analysis of stiffness elements is given. Furthermore, the minimum and maximum rotation/translation stiffness are introduced to analyze the effect of the structural parameters of continuum robot on the overall rotation and translation stiffness. Finally, the experiments are used to validate the proposed stiffness model. The experimental results show that the proposed stiffness model of continuum robot is correct and the errors are less than $7 \%$.
\end{abstract}

\section{Introduction}

Continuum robot is a new kind of bionic robot, inspired by elephant trunks and octopus tentacles. Continuum robot does not have its own joints, which can produce flexible deformation in any part, so it has a strong ability to avoid obstacles and better adapt to the complex unstructured environments. Continuum robots offer a number of potential advantages over the traditional rigid-link robots in applications involving disaster relief [1], industrial applications [2], and medical aid [3].

The main types of continuum robots include the roddriven continuum robot [4], cable-driven continuum robot [5], pneumatic continuum robot [6], and concentric tube continuum robot [7]. Du et al. [8] developed an optimization notched continuum manipulator based on performance evaluation indices. Tian et al. [9] proposed the kinematic analysis of a continuum bionic robot with three flexible actuation rods. Moreover, Bergeles et al. [10] presented optimization framework based on anatomical and surgical task constraints. Li et al. [11] developed a new constrained wire-driven flexible mechanism which has a larger workspace and is more dexterous compared to the existing surgical arms. He et al. [12] proposed a multibackbone continuum robot which is driven by NiTi alloy wire. Trivedi et al. [13] discussed the novel capabilities of soft robots, described examples from nature that provide biological inspiration, surveyed the state of the art, and outlined existing challenges in soft robot design, modeling, fabrication, and control. Webster III and Jones [14] reviewed several modeling approaches in a common frame and notational convention, illustrating that, for piecewise constant curvature, they produce identical results.

The analysis of stiffness is an important step in the design and control of continuum robots, since it determines the relationship between the deformation and the force of continuum robots. Selig and Ding [15] applied the screw theory [16] to analyze compliance and stiffness matrices of a beam. Pei et al. [17] studied the compliance of cartwheel flexural hinges. Ding and Dai [18] investigated spatial continuous compliance on both serial and parallel mechanisms based on screw theory and Lie groups, applying eigenvectors and eigenvalues to identify principal screws in the mechanisms. Awtar and Sen [19] proposed a generalized constraint model for compliance and stiffness analysis of 2D beam flexures. Krishnan et al. [20] studied serial and parallel concatenation of building blocks. Tunay [21] proposed a concept of equivalent bending stiffness 
to establish the kinematic model of continuum robot. Gao et al. [22] established a mathematical model for predicting the loaded posture of a single section continuum manipulator. Qi et al. [23] analyzed the compliance characteristics of a new planar spring continuum robot. Gravagne et al. [24] discussed the dynamics of a planar continuum backbone section, incorporating a large-deflection dynamic model. Trivedi et al. [25] presented a new approach for modeling soft robotic manipulators that incorporates the effect of material nonlinearities and distributed weight and payload. Camarillo et al. [26] proposed a new linear model for transforming desired beam configuration to tendon displacements and vice versa. Fras et al. [27] described the design and implementation of a static model used for position estimation of a flexible modular medical manipulator equipped with optic-fiber based sensors. Till and Rucker [28] exhibited low numerical damping, handled arbitrarily large time steps, and provided an accurate, high-order representation of the rod shape in steady state. Hadi Sadati et al. [29] introduced a novel series solution for variable-curvature Cosserat rod static and Lagrangian dynamic method.

Several different performance indices have been proposed for stiffness evaluation, including determinant of stiffness matrix, average stiffness, and minimum and maximum stiffness [30, 31]. The determinant of stiffness matrix does not distinguish specific stiffness values, and the average stiffness cannot give enough information of stiffness values [32]. The minimum and maximum stiffness are minimum and maximum eigenvalue of stiffness matrix, respectively, which can indicate variation range of stiffness values, and the corresponding eigenvector directions represent the minimum and maximum stiffness directions, respectively. However, it is well known that the conventional minimum and maximum stiffness cannot be applied to a $6 \times 6$ symmetric stiffness/compliance matrix. This is due to the fact that the eigenvalues of a stiffness/compliance matrix are not consistent unit and no way to compare the sizes of eigenvalues. As a consequence, we define the minimum and maximum rotation/translation stiffness, to evaluate the influence of the structural parameters of continuum robot on the rotation/translation stiffness of continuum robot.

In the paper, a general loading is represented by a wrench, while a general deformation is represented by a twist. The main contribution is to present a method to establish the stiffness model of a single section multibackbone continuum robot based on screw theory and Euler-Bernoulli beam, as well as analyzing the effect of the structural parameters of continuum robot on the overall rotation and translation stiffness by the minimum and maximum rotation/translation stiffness. The remainder of the paper is organized as follows: In Section 2, we give structure overview of a single section multibackbone continuum robot. In Section 3, based on the screw theory and Euler-Bernoulli beam, a brief stiffness model which illustrates the end configuration of the continuum robot under external load is deduced. In Section 4 , the stiffness elements are analyzed to fully indicate relationship between wrench and twist of continuum robot and obtain the influence of the structural parameters of continuum robot on the stiffness elements. In addition, the minimum

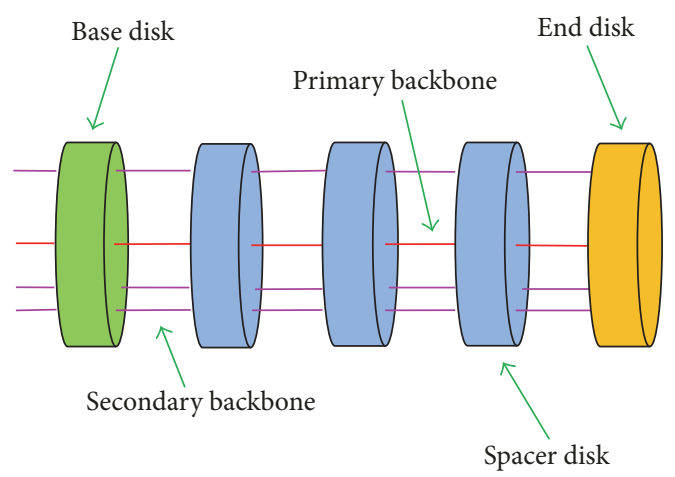

FIGURE 1: The simplified structure of a single section multibackbone continuum robot.

and maximum rotation/translation stiffness are introduced to evaluate the effect of the structural parameters of continuum robot on the overall rotation and translation stiffness. In Section 5 , the proposed stiffness model is validated by the experiments. The experimental results indicate that proposed stiffness model of continuum robot is correct. In Section 6, some discussions are given. In Section 7, conclusions are provided.

\section{Overview of a Single Section Multibackbone Continuum Robot}

The simplified structure of a single section multibackbone continuum robot is shown in Figure 1. The continuum robot is composed of a base disk, several spacer disks, an end disk, and four super elastic NiTi wires as its backbones. The central NiTi wire is the primary backbone and the remaining NiTi wires are the secondary backbones. The primary backbone is attached to all disks, and all disks are distributed in equal distance. The secondary backbones are equidistant from primary backbone and from each other. The secondary backbones are only attached to the end disk and slide through holes in base disk and spacer disks, which have double effect: driving robot to achieve two degrees of freedom bending motion and providing auxiliary support to increase the stiffness of continuum robot.

\section{Stiffness Model of Continuum Robot}

In order to facilitate the derivation of the presented theory, the model assumptions of continuum robot are summarized in this paper as follows:

(1) The disks of robot are thin, so the thickness of the disks is ignored.

(2) The friction between the disks and backbones is neglected.

(3) The continuum robot is under static balance.

(4) The gravity of all the disks and the four backbones, which is ignored, is very smaller.

(5) We assume that the deformation of backbones is sufficiently small so that the principle of Euler-Bernoulli beam theory applies. 


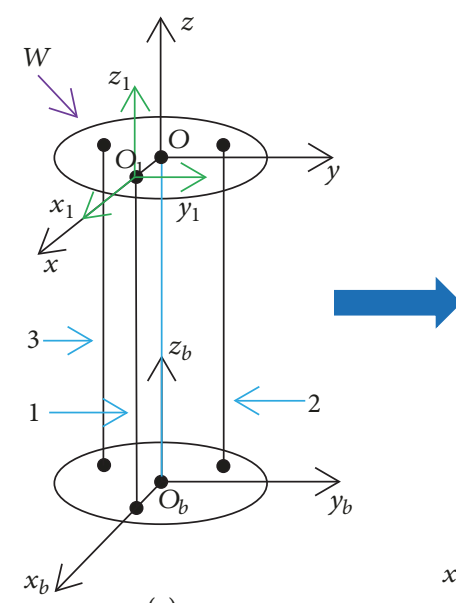

(a)

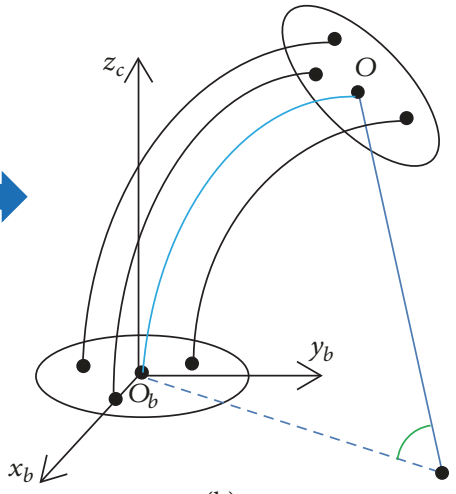

(b)

FIgURE 2: The continuum robot is subjected to the external load at the end of continuum robot.

When the continuum robot is not exerted by an external load and the secondary backbone does not pull and push the continuum robot, then the simplified geometric configuration of continuum robot is shown in Figure 2(a) and the length of all the backbones are equal. The global coordinate system $O-x y z$ is placed at the end disk, as we are interested in the motion of the end of continuum robot. Point $O$ is geometric center of end disk, the axis $x$ points from point $O$ to the first secondary backbone, the axis $z$ is perpendicular to the end disk, and the axis $y$ is then defined according to the right-hand rule. Moreover, the coordinate system $O_{b}-x_{b} y_{b} z_{b}$ is established at point $O_{b}$, the axis $x_{b}$ is parallel to the axis $x$, the axis $y_{b}$ is parallel to the axis $y$, and the axis $z_{b}$ is parallel to the axis $z$. The secondary backbones are the average interval 120 degrees around the primary backbone. Assuming that the continuum robot is subjected to the external load at the end of continuum robot, the configuration of continuum robot is shown in Figure 2(b).

For easy comprehension of the deduction of the presented theory, in this paper, symbols are defined as follows:

$i$ : the index of secondary backbones $i=1,2,3$.

$r$ : the distance from the primary backbone to each secondary backbone on the disk.

$L$ : the length of primary backbone.

$L_{i}$ : the length of secondary backbones $i=1,2,3$.

$r_{b}$ : the radius of backbones.

In the framework of a screw theory [14] and the global coordinate system $O-x y z$, a small deformation of continuum robot is defined as a twist $\xi=(\phi, \delta)^{T}$ in axis coordinates. $\xi$ is an element of Lie algebra se(3) of Lie group $\mathrm{SE}(3)$, where $\phi=\left(\phi_{x}, \phi_{y}, \phi_{z}\right)^{T}$ represents the corresponding rotational deflections around their corresponding axes and $\delta=\left(\delta_{x}, \delta_{y}, \delta_{z}\right)^{T}$ reveals the three translational deflections along their corresponding axes. $W=(\tau, f)^{T}$ is defined as the wrench at the end of the continuum robot in ray coordinates, which is an element of the dual space of Lie algebra $\mathrm{se}^{*}(3)$, where $\tau=\left(\tau_{x}, \tau_{y}, \tau_{z}\right)^{T}$ represents three moments around the axes $x, y$, and $z$, respectively; $f=\left(f_{x}, f_{y}, f_{z}\right)^{T}$ reveals three forces along axes $x, y$, and $z$, respectively. According to the elastic theory, wrench $W$ and twist $\xi$ are related by

$$
W=K_{P} \xi, \quad \xi=C_{P} W, K_{P}=C_{P}^{-1},
$$

where $K_{P}$ and $C_{P}$ are the stiffness and compliance matrices of continuum robot in the global coordinate system $O-x y z$, respectively.

In the following, we derive stiffness model of continuum robot. First, the compliance matrix of primary backbone in the global coordinate system $O-x y z$ can be derived by screw theory [17] and Euler-Bernoulli beam [13], which is expressed as

$$
C_{0}=\left[\begin{array}{cccccc}
\frac{L}{E I_{x}} & 0 & 0 & 0 & -\frac{L^{2}}{2 E I_{x}} & 0 \\
0 & \frac{L}{E I_{y}} & 0 & \frac{L^{2}}{2 E I_{y}} & 0 & 0 \\
0 & 0 & \frac{L}{G J} & 0 & 0 & 0 \\
0 & \frac{L^{2}}{2 E I_{y}} & 0 & \frac{L^{3}}{3 E I_{y}} & 0 & 0 \\
-\frac{L^{2}}{2 E I_{x}} & 0 & 0 & 0 & \frac{L^{3}}{3 E I_{x}} & 0 \\
0 & 0 & 0 & 0 & 0 & \frac{L}{E A}
\end{array}\right],
$$

where $A=\pi r_{b}^{2}$ is the area of the cross section, $I_{x}=(1 / 4) \pi r_{b}^{4}$ and $I_{y}=(1 / 4) \pi r_{b}^{4}$ are the area moments, $J=(1 / 2) \pi r_{b}^{4}$ is the torsional moment of inertia, $E$ denotes the elastic module of the material, and $G$ denotes the shear module of the material.

For convenience, by extracting common factor, (2) is simplified as 


$$
C_{0}=\frac{L}{E I_{x}}\left[\begin{array}{cccccc}
1 & 0 & 0 & 0 & -\frac{L}{2} & 0 \\
0 & 1 & 0 & \frac{L}{2} & 0 & 0 \\
0 & 0 & \frac{1}{2 \mu} & 0 & 0 & 0 \\
0 & \frac{L}{2} & 0 & \frac{L^{2}}{3} & 0 & 0 \\
-\frac{L}{2} & 0 & 0 & 0 & \frac{L^{2}}{3} & 0 \\
0 & 0 & 0 & 0 & 0 & \frac{r_{b}^{2}}{4}
\end{array}\right],
$$

where

$$
\mu=\frac{G}{E}=\frac{1}{2(1+v)}
$$

and $v$ is the Poisson's ratio.

It is assumed that the $K_{0}$ is stiffness matrix of primary backbone in the global coordinate system $O-x y z$, and then the stiffness matrix $K_{0}$ is calculated as

$$
K_{0}=C_{0}^{-1}
$$

The local coordinate system $O_{i}-x_{i} y_{i} z_{i}$ is established for the $i(i=1,2,3)$ th secondary backbone at the end disk, as shown in Figure 2(a). Point $O_{i}$ is the intersection point of the $i$ th secondary backbone and the end disk, the axis $x_{i}$ is parallel to axis $x$, the axis $y_{i}$ is parallel to axis $y$, and the axis $z_{i}$ is parallel to axis $z$. Suppose that $C_{s i}$ is the compliance matrix of the $i$ th secondary backbone in the local coordinate system $O_{i}-x_{i} y_{i} z_{i}$, since $L_{i}=L(i=1,2,3)$, which can be obtained as

$$
C_{s i}=\frac{L}{E I_{x}}\left[\begin{array}{cccccc}
1 & 0 & 0 & 0 & -\frac{L}{2} & 0 \\
0 & 1 & 0 & \frac{L}{2} & 0 & 0 \\
0 & 0 & \frac{1}{2 \mu} & 0 & 0 & 0 \\
0 & \frac{L}{2} & 0 & \frac{L^{2}}{3} & 0 & 0 \\
-\frac{L}{2} & 0 & 0 & 0 & \frac{L^{2}}{3} & 0 \\
0 & 0 & 0 & 0 & 0 & \frac{r_{b}^{2}}{4}
\end{array}\right] \text {, }
$$

where $i=1,2,3$.

Since compliance matrix $C_{s i}(i=1,2,3)$ is represented in the different local coordinate system, in order to establish the stiffness model of continuum robot, we should transform the compliance matrix $C_{s i}(i=1,2,3)$ to the global coordinate system by coordinate transformation. Here, to shift the local coordinate system $O_{i}-x_{i} y_{i} z_{i}$ of each secondary backbone into the global coordinate system $O-x y z$, an adjoint action of Lie group SE(3) on its Lie algebra is introduced by a $6 \times 6$ matrix representation [21]:

$$
\operatorname{Ad}_{g}=\left[\begin{array}{cc}
R & 0 \\
D R & R
\end{array}\right]
$$

where $R$ is a $3 \times 3$ rotation matrix representing the orientation of the local coordinate system $O_{i}-x_{i} y_{i} z_{i}$, relative to the global coordinate system $O-x y z$; for instance, the 1st secondary backbone $R$ is a unit matrix. $D$ is a skew-symmetric matrix spanned by the position vector $d$ from the origin of frame $O-x y z$ to the origin of frame $O_{i}-x_{i} y_{i} z_{i}$; for example, the 1st secondary backbone $d=[r, 0,0]$.

Here, the adjoint transformation matrix of the $i$ th $(i=$ $1,2,3)$ secondary backbone is given by

$$
\operatorname{Ad}_{g i}=\left[\begin{array}{cc}
I & 0 \\
D_{i} I & I
\end{array}\right], \quad \operatorname{Ad}_{g i} \in \operatorname{Se}(3),
$$

where

$$
\begin{aligned}
I & =\left[\begin{array}{lll}
1 & 0 & 0 \\
0 & 1 & 0 \\
0 & 0 & 1
\end{array}\right], \\
D_{1} & =\left[\begin{array}{lll}
0 & 0 & 0 \\
0 & 0 & -r \\
0 & r & 0
\end{array}\right], \\
D_{2} & =\left[\begin{array}{ccc}
0 & 0 & \frac{\sqrt{2}}{2} r \\
0 & 0 & \frac{\sqrt{2}}{2} r \\
-\frac{\sqrt{2}}{2} r & -\frac{\sqrt{2}}{2} r & 0
\end{array}\right], \\
D_{3}= & {\left[\begin{array}{ccc}
0 & 0 & -\frac{\sqrt{2}}{2} r \\
0 & 0 & \frac{\sqrt{2}}{2} r \\
\frac{\sqrt{2}}{2} r & -\frac{\sqrt{2}}{2} r & 0
\end{array}\right] . }
\end{aligned}
$$

In addition, the transpose of the adjoint transformation matrix is given by

$$
\operatorname{Ad}_{g i}^{T}=\left[\begin{array}{cc}
I & -I^{T} D_{i} \\
0 & I
\end{array}\right] .
$$

It is supposed that $C_{i}$ is the compliance matrix of the $i$ th secondary backbone in the global coordinate system $O-x y z$ and $\xi_{i}^{\prime}$ and $W_{i}^{\prime}$ are twist and wrench at the end of the $i$ th secondary backbone in the global coordinate system $O-x y z$, respectively. $\xi_{i}$ and $W_{i}$ are twist and wrench at the end of the $i$ th secondary backbone in the local coordinate system $\mathrm{O}_{i}-x_{i} y_{i} z_{i}$, yielding

$$
\begin{aligned}
& \xi_{i}^{\prime}=C_{i} W_{i}^{\prime}, \\
& \xi_{i}=C_{s i} W_{i} .
\end{aligned}
$$

Moreover, the coordinates of a twist and a wrench in the global coordinate system $O-x y z$ are calculated as [16]

$$
\begin{gathered}
\xi_{i}^{\prime}=\operatorname{Ad}_{g i} \xi_{i}, \\
W_{i}^{\prime}=\operatorname{Ad}_{g i}^{-T} W_{i} .
\end{gathered}
$$


To obtain the compliance matrix $C_{i}$ in the global coordinate system $O-x y z$, we deduct it as follows based on (11) and (12):

$$
\xi_{i}^{\prime}=\operatorname{Ad}_{g i} \xi_{i}=\operatorname{Ad}_{g i} C_{s i} W_{i}=\operatorname{Ad}_{g i} C_{s i} \operatorname{Ad}_{g i}^{T} W_{i}^{\prime}
$$

Accordingly, the compliance matrix $C_{i}$ can be obtained according to the relation

$$
C_{i}=\operatorname{Ad}_{g i} C_{s i} \operatorname{Ad}_{g i}^{T},
$$

where $i=1,2,3$.

Similarly, we can derive the stiffness matrix $K_{i}$ of the $i$ ( $i=$ $1,2,3)$ th secondary backbone in the global coordinate system $O-x y z$ as

$$
K_{i}=C_{i}^{-1}=\left(\operatorname{Ad}_{g i} C_{s i} \operatorname{Ad}_{g i}^{T}\right)^{-1}
$$

Since the continuum robot is composed of four NiTi wires in parallel, if all deformations are presented into the same global coordinate system $O-x y z$, then the overall stiffness matrix of the parallel flexible mechanism is the sum of the stiffness matrix of each flexible mechanism [16]; the overall stiffness matrix $K_{P}$ of continuum robot can be obtained as

$$
K_{P}=\sum_{i=0}^{3} K_{i},
$$

where

$$
\begin{aligned}
& K_{0}=\frac{E I_{x}}{L}\left[\begin{array}{cccccc}
4 & 0 & 0 & 0 & \frac{6}{L} & 0 \\
0 & 4 & 0 & -\frac{6}{L} & 0 & 0 \\
0 & 0 & 2 \mu & 0 & 0 & 0 \\
0 & -\frac{6}{L} & 0 & \frac{12}{L^{2}} & 0 & 0 \\
\frac{6}{L} & 0 & 0 & 0 & \frac{12}{L^{2}} & 0 \\
0 & 0 & 0 & 0 & 0 & \frac{4}{r_{b}^{2}}
\end{array}\right] \text {, }
\end{aligned}
$$

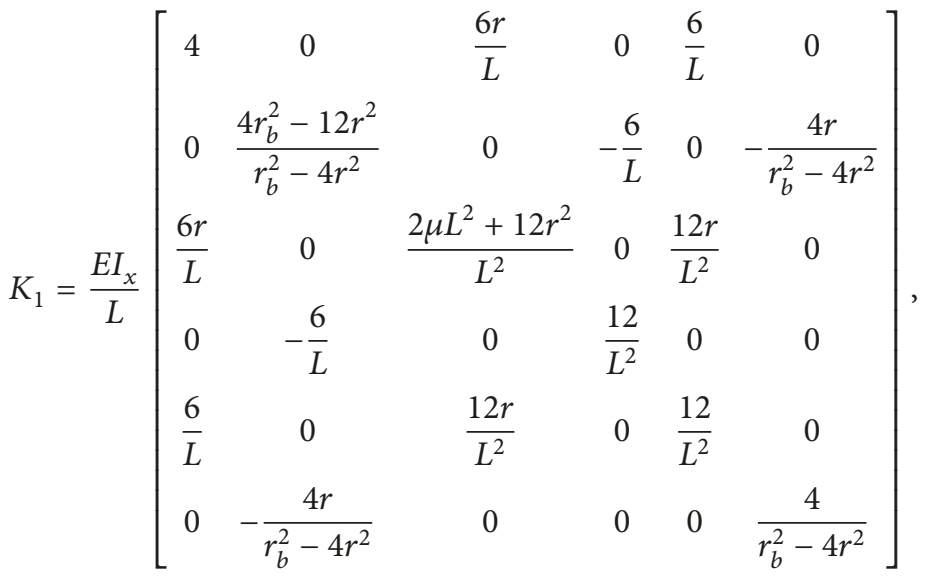

$$
\begin{aligned}
& K_{2}=\frac{E I_{x}}{L}\left[\begin{array}{cccccc}
\frac{4 r_{b}^{2}+2 r^{2}}{r_{b}^{2}} & \frac{2 r^{2}}{r_{b}^{2}} & -\frac{3 \sqrt{2} r}{L} & 0 & \frac{6}{L} & \frac{2 \sqrt{2} r}{r_{b}^{2}} \\
\frac{2 r^{2}}{r_{b}^{2}} & \frac{4 r_{b}^{2}+2 r^{2}}{r_{b}^{2}} & \frac{3 \sqrt{2} r}{L} & -\frac{6}{L} & 0 & \frac{2 \sqrt{2} r}{r_{b}^{2}} \\
-\frac{3 \sqrt{2} r}{L} & \frac{3 \sqrt{2} r}{L} & \frac{2 \mu L^{2}+12 r^{2}}{L^{2}} & -\frac{6 \sqrt{2} r}{L^{2}} & -\frac{6 \sqrt{2} r}{L^{2}} & 0 \\
0 & -\frac{6}{L} & -\frac{6 \sqrt{2} r}{L^{2}} & \frac{12}{L^{2}} & 0 & 0 \\
\frac{6}{L} & 0 & -\frac{6 \sqrt{2} r}{L^{2}} & 0 & \frac{12}{L^{2}} & 0 \\
\frac{2 \sqrt{2} r}{r_{b}^{2}} & \frac{2 \sqrt{2} r}{r_{b}^{2}} & 0 & 0 & 0 & \frac{4}{r_{b}^{2}}
\end{array}\right] \text {, }
\end{aligned}
$$




$$
K_{3}=\frac{E I_{x}}{L}\left[\begin{array}{cccccc}
\frac{4 r_{b}^{2}+2 r^{2}}{r_{b}^{2}} & -\frac{2 r^{2}}{r_{b}^{2}} & -\frac{3 \sqrt{2} r}{L} & 0 & \frac{6}{L} & -\frac{2 \sqrt{2} r}{r_{b}^{2}} \\
-\frac{2 r^{2}}{r_{b}^{2}} & \frac{4 r_{b}^{2}+2 r^{2}}{r_{b}^{2}} & -\frac{3 \sqrt{2} r}{L} & -\frac{6}{L} & 0 & \frac{2 \sqrt{2} r}{r_{b}^{2}} \\
-\frac{3 \sqrt{2} r}{L} & -\frac{3 \sqrt{2} r}{L} & \frac{2 \mu L^{2}+12 r^{2}}{L^{2}} & \frac{6 \sqrt{2} r}{L^{2}} & -\frac{6 \sqrt{2} r}{L^{2}} & 0 \\
0 & -\frac{6}{L} & \frac{6 \sqrt{2} r}{L^{2}} & \frac{12}{L^{2}} & 0 & 0 \\
\frac{6}{L} & 0 & -\frac{6 \sqrt{2} r}{L^{2}} & 0 & \frac{12}{L^{2}} & 0 \\
-\frac{2 \sqrt{2} r}{r_{b}^{2}} & \frac{2 \sqrt{2} r}{r_{b}^{2}} & 0 & 0 & 0 & \frac{4}{r_{b}^{2}}
\end{array}\right] .
$$

Substituting (17) into (16) yields

$$
K_{P}=\left[k_{p i j}\right]_{6 \times 6}=\frac{E I_{x}}{L}\left[\begin{array}{cccccc}
k_{11} & 0 & k_{13} & 0 & k_{15} & 0 \\
0 & k_{22} & 0 & k_{24} & 0 & k_{26} \\
k_{31} & 0 & k_{33} & 0 & k_{35} & 0 \\
0 & k_{42} & 0 & k_{44} & 0 & 0 \\
k_{51} & 0 & k_{53} & 0 & k_{55} & 0 \\
0 & k_{62} & 0 & 0 & 0 & k_{66}
\end{array}\right] \text {, }
$$

where $i=1,2,3,4,5,6 ; j=1,2,3,4,5,6$; and

$$
\begin{aligned}
& k_{11}=16+\frac{4 r^{2}}{r_{b}^{2}}, \\
& k_{13}=k_{31}=\frac{6(1-\sqrt{2}) r}{L} \\
& k_{15}=k_{51}=\frac{24}{L}, \\
& k_{22}=16+\frac{8 r^{2}\left(2 r^{2}-r_{b}^{2}\right)}{r_{b}^{2}\left(4 r^{2}-r_{b}^{2}\right)} \\
& k_{24}=k_{42}=-\frac{24}{L}, \\
& k_{26}=k_{62}=\frac{4 \sqrt{2} r}{r_{b}^{2}}+\frac{4 r}{4 r^{2}-r_{b}^{2}} \\
& k_{66}=\frac{12}{r_{b}^{2}}-\frac{4}{4 r^{2}-r_{b}^{2}} . \\
& k_{44}=k_{55}=\frac{48}{L^{2}}, \\
& k^{2}, \\
& k_{53}=\frac{36 r^{2}}{12(1-\sqrt{2}) r} \\
& L^{2}
\end{aligned}
$$

It can be seen that $K_{P}$ in (18) is determined by six independent design parameters, $E, I_{x}, L, r, r_{b}$, and $\mu$. Simultaneously, the stiffness matrix $K_{P}$ gives the relationship between the wrench and twist at the end of the continuum robot. Furthermore, the compliance matrix is the inverse of the stiffness matrix which is calculated as

$$
C_{P}=\frac{L}{E I_{x}}\left[\begin{array}{cccccc}
c_{11} & 0 & 0 & 0 & c_{15} & 0 \\
0 & c_{22} & 0 & c_{24} & 0 & c_{26} \\
0 & 0 & c_{33} & 0 & c_{35} & 0 \\
0 & c_{42} & 0 & c_{44} & 0 & c_{46} \\
c_{51} & 0 & c_{53} & 0 & c_{55} & 0 \\
0 & c_{62} & 0 & c_{64} & 0 & c_{66}
\end{array}\right]
$$

where

$$
\begin{gathered}
c_{11}=\Gamma, \\
c_{15}=c_{51}=-\frac{L \Gamma}{2} \\
c_{22}=\frac{1.2 r^{2} r_{b}^{2}-4 r_{b}^{4}}{\sigma} \times 10^{5} \\
c_{24}=c_{42}=\frac{2 L r_{b}^{2}\left(3 r^{2}-r_{b}^{2}\right)}{\sigma} \times 10^{5} \\
c_{26}=2 c_{46}=2 c_{64}=\frac{125 r_{b}^{2} r\left(313 r_{b}^{2}-4452 r^{2}\right)}{\sigma} \\
c_{33}=\frac{2.5 L^{2}}{g} \times 10^{5}, \\
c_{35}=c_{53}=\frac{25875 L^{2} r}{g}, \\
c_{55}=\frac{\frac{q}{48 g}}{48 g},
\end{gathered}
$$




$$
\begin{gathered}
c_{66}=\frac{s}{\sigma}, \\
\Gamma=\frac{r_{b}^{2}}{4\left(r^{2}+r_{b}^{2}\right)} \\
\sigma=\left(1.665 r^{2} r_{b}^{2}-1.6 r_{b}^{4}+1.652 r^{4}\right) \times 10^{6} \\
g=\left(2 \mu L^{2}-8.871 r^{2}\right) \times 10^{6} \\
q=L^{2}\left(16.065 r^{2} r_{b}^{2}-6.4 r_{b}^{4}+1.652 r^{4}\right) \times 10^{6} \\
e=L^{2}\left(8 \mu L^{2} r_{b}^{2}+2 \mu L^{2} r^{2}+35.614 r^{2} r_{b}^{2}+9 r^{4}\right) \times 10^{6} \\
s=\left(2 r^{2} r_{b}^{4}-r_{b}^{6}+4 r^{4} r_{b}^{2}\right) \times 10^{5} .
\end{gathered}
$$

\section{Stiffness Analysis of Continuum Robot}

Based on the stiffness model of continuum robot in Section 3, we will first analyze the effect of the structural parameters of continuum robot on the stiffness elements in this section. Then, the influence of the structural parameters on the overall rotation and translation stiffness is analyzed. Finally, to more intuitively understand the stiffness elements of continuum robot, the stiffness elements are analyzed by the numerical example.

4.1. Analysis of Stiffness Elements. Each element in the stiffness matrix reflects the mapping relationship between each element in the wrench and each element in the twist. Hence, through the analysis of the stiffness elements of stiffness matrix $K_{P}$ in (18), we can obtain the following conclusions.

(1) The rotational stiffness around axes $x, y$, and $z$ is calculated as

$$
\begin{aligned}
& k_{P 11}=\frac{\tau_{x}}{\phi_{x}}=\frac{E I_{x}}{L} k_{11}=\frac{\pi E r_{b}^{2}\left(4 r_{b}^{2}+r^{2}\right)}{L} \\
& k_{P 22}=\frac{\tau_{y}}{\phi_{y}}=\frac{E I_{x}}{L} k_{22}=\frac{\pi E r_{b}^{2}\left(4 r^{4}+14 r^{2} r_{b}^{2}-4 r_{b}^{4}\right)}{L\left(4 r^{2}-r_{b}^{2}\right)} \\
& k_{P 33}=\frac{\tau_{z}}{\phi_{z}}=\frac{E I_{x}}{L} k_{33}=\frac{\pi E r_{b}^{4}\left(2 \mu L^{2}+9 r^{2}\right)}{L^{3}}
\end{aligned}
$$

By (22), (23), and (24), assuming that $r$ and $r_{b}$ are constant values, it can be seen that when length $L$ increases, with a decreasing of the rotational stiffness $k_{P 11}, k_{P 22}$, and $k_{P 33}$, obviously, $k_{P 11}$ and $k_{P 22}$ are inversely proportional to $L$. By (22) and (24), supposing that $L$ and $r$ are constant values, when length $r_{b}$ increases, $k_{P 11}$ and $k_{P 33}$ also increase. Assuming that $L$ and $r_{b}$ are constant values, when length $r$ increases, $k_{P 11}$ and $k_{P 33}$ also increase. By (23), assuming that $L$ and $r_{b}$ are constant values, when length $r$ increases, $k_{P 22}$ also increases, as shown Figure 3.

(2) The translational stiffness along axes $x, y$, and $z$ is expressed as

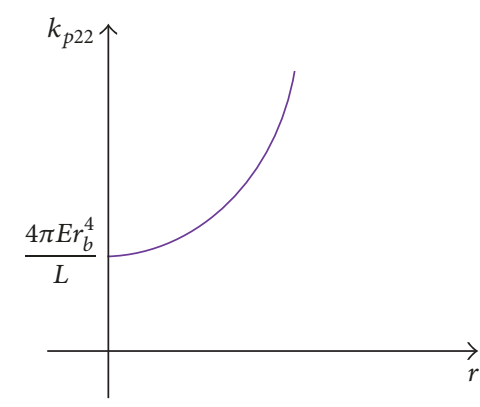

FIGURE 3: Relationship between $k_{P 22}$ and $r$.

$$
\begin{aligned}
& k_{P 44}=\frac{f_{x}}{\delta_{x}}=\frac{E I_{x}}{L} k_{44}=\frac{12 \pi E r_{b}^{4}}{L^{3}} \\
& k_{P 55}=\frac{f_{y}}{\delta_{y}}=\frac{E I_{x}}{L} k_{55}=\frac{12 \pi E r_{b}^{4}}{L^{3}} \\
& k_{P 66}=\frac{f_{z}}{\delta_{z}}=\frac{E I_{x}}{L} k_{66}=\frac{4 \pi E r_{b}^{2}\left(3 r^{2}-r_{b}^{2}\right)}{L\left(4 r^{2}-r_{b}^{2}\right)} .
\end{aligned}
$$

By (25) and (26), changing $r$ has no effect on the $k_{P 44}$ and $k_{P 55}$. Assuming that $r_{b}$ is constant value, when length $L$ increases, with a decreasing of the translational stiffness $k_{P 44}$ and $k_{P 55}$, which are inversely proportional to $L^{3}$. Assume that $r_{b}$ is constant value, when length $L$ increases, with decreasing of the translational stiffness $k_{P 44}$ and $k_{P 55}$, which are inversely proportional to $L^{3}$. Supposing that the length $L$ is constant value, when length $r_{b}$ increases, $k_{P 44}$ and $k_{P 55}$ also increase, which are directly proportional to $r_{b}^{4}$. By (27), assuming that $r$ and $r_{b}$ are constant values, it can be seen that when length $L$ increases, but $k_{P 66}$ decreases, obviously, $k_{P 66}$ is inversely proportional to $L$. Supposing that $L$ and $r_{b}$ are constant values, when length $r$ increases, $k_{P 66}$ decreases. By (18), the translational stiffness of $k_{P 44}, k_{P 55}$, and $k_{P 66}$ is decoupled; that is, the forces $f_{x}, f_{y}$, and $f_{z}$ only produce translation along axes $x, y$, and $z$, respectively.

(3) The rotational stiffness which is produced by force $f_{x}$ is calculated as

$$
k_{P 42}=\frac{f_{x}}{\phi_{y}}=\frac{E I_{x}}{L} k_{42}=-\frac{6 \pi E r_{b}^{4}}{L^{2}} .
$$

By (28), $f_{x}$ only produce rotation around axis $y$. Dividing (25) by (28) and taking the absolute value, which is defined as $\varepsilon_{1}$, yield

$$
\varepsilon_{1}=\left|\frac{k_{P 44}}{k_{P 42}}\right|=\left|\frac{\phi_{y}}{\delta_{x}}\right|=\frac{2}{L} .
$$

By (29), if length $L$ increases, $\varepsilon_{1}$ decreases. The result shows that when the force $f_{x}$ is a constant value and length $L$ increases, the value $\left|\phi_{y} / \delta_{x}\right|$ decreases. 
(4) The rotational stiffness which is produced by force $f_{y}$ is calculated as

$$
\begin{aligned}
& k_{P 51}=\frac{f_{y}}{\phi_{x}}=\frac{E I_{x}}{L} k_{51}=\frac{6 \pi E r_{b}^{4}}{L^{2}} \\
& k_{P 53}=\frac{f_{y}}{\phi_{z}}=\frac{E I_{x}}{L} k_{53}=\frac{3(1-\sqrt{2}) \pi E r_{b}^{4} r}{L^{3}} .
\end{aligned}
$$

By (30) and (31), $f_{y}$ produce two rotational stiffness elements. One is the rotation stiffness $k_{P 51}$ around the axis $x$, which is a function of the variables $L$ and $r_{b}$, The effects of variables $L$ and $r_{b}$ on $k_{P 51}$ can be easily obtained by (30). The other is the rotational stiffness $k_{P 53}$ around the axis $z$, which is a function of the variables $L, r$, and $r_{b}$, The effects of variables $L, r$, and $r_{b}$ on $k_{P 53}$ can be easily given by (31). Equation (26) is divided by (30), which is defined as $\varepsilon_{2}$. Equation (26) is divided by (31), taking the absolute value, which is defined as $\varepsilon_{3}$. Equation (31) is divided by (30), taking the absolute value, which is defined as $\varepsilon_{4}$. This yields

$$
\begin{aligned}
& \varepsilon_{2}=\frac{k_{P 55}}{k_{P 51}}=\frac{\phi_{x}}{\delta_{y}}=\frac{2}{L} \\
& \varepsilon_{3}=\left|\frac{k_{P 55}}{k_{P 53}}\right|=\left|\frac{\phi_{z}}{\delta_{y}}\right|=\frac{4(1+\sqrt{2})}{r} \\
& \varepsilon_{4}=\left|\frac{k_{P 53}}{k_{P 51}}\right|=\left|\frac{\phi_{x}}{\phi_{z}}\right|=\frac{(\sqrt{2}-1) r}{2 L} .
\end{aligned}
$$

By (32), when length $L$ increases, $\varepsilon_{2}$ decreases. The result explains that when the force $f_{y}$ is a constant value and length $L$ increases, the value $\phi_{x} / \delta_{y}$ decreases. By (33), when length $r$ increases, $\varepsilon_{3}$ decreases, indicating that when the force $f_{y}$ is a constant value and length $r$ increases, the value $\left|\phi_{z} / \delta_{y}\right|$ decreases. By (34), assuming that $L$ is constant value, it can be seen that when $r$ increases, $\varepsilon_{4}$ also increases. The result indicates that when $f_{y}, L$ are constant values and $r$ increases, the value $\left|\phi_{x} / \phi_{z}\right|$ increases. Supposing that $r$ is constant value, when $L$ increases, $\varepsilon_{5}$ also decreases. Indicating that when $f_{y}$, $r$ are a constant values and $L$ increases, the value $\left|\phi_{x} / \phi_{z}\right|$ decreases.

(5) The rotational stiffness which is produced by force $f_{z}$ is calculated as

$$
\begin{aligned}
k_{P 62} & =\frac{f_{z}}{\phi_{y}}=\frac{E I_{x}}{L} k_{62} \\
& =\frac{\pi E r_{b}^{2} r\left(4 \sqrt{2} r^{2}-(\sqrt{2}-1) r_{b}^{2}\right)}{L\left(4 r^{2}-r_{b}^{2}\right)} .
\end{aligned}
$$

Dividing (27) by (35), which is defined as $\varepsilon_{5}$, we obtain

$$
\varepsilon_{5}=\frac{k_{P 66}}{k_{P 62}}=\frac{\phi_{y}}{\delta_{z}}=\frac{4\left(3 r^{2}-r_{b}^{2}\right)}{r\left(4 \sqrt{2} r^{2}-(\sqrt{2}-1) r_{b}^{2}\right)} \text {. }
$$

When $r_{b} \ll r$, (36) is simplified as

$$
\varepsilon_{5}=\frac{k_{P 66}}{k_{P 62}} \approx \frac{3}{\sqrt{2} r} .
$$

By (37), when $r_{b} \ll r$ and $r$ increases, $\varepsilon_{5}$ decreases. The result explains that when the force $f_{z}$ is a constant value, $r_{b} \ll$ $r$, and $r$ increases, the value $\phi_{y} / \delta_{z}$ decreases. When $r \ll r_{b}$, (36) is simplified as

$$
\varepsilon_{5}=\frac{k_{P 66}}{k_{P 62}} \longrightarrow+\infty
$$

By (38), when $r \ll r_{b}, \varepsilon_{5}$ tends to positive infinity. The result indicates that when the force $f_{z}$ is a constant value and $r \ll r_{b}$, the value $\phi_{y} / \delta_{z}$ tends to positive infinity.

4.2. Eigen-Stiffness Analysis of Continuum Robot. Since the eigenvalues of the stiffness matrix in (18) are not consistent unit, there is no way to compare the sizes of eigenvalues; the conventional minimum/maximum stiffness cannot be applied to a $6 \times 6$ symmetric stiffness/compliance matrix. Here, we define the minimum and maximum rotation/translation stiffness to evaluate the effect of the structural parameters of continuum robot on the rotation/translation stiffness. In order to facilitate the presentation of this definition, we first block the stiffness matrix $K_{P}$ in (18), which can be generally represented in the form of

$$
K_{P}=\left[\begin{array}{cc}
A & B \\
B^{T} & C
\end{array}\right]
$$

where the symmetric $3 \times 3$ block matrices $A$ and $C$ denote the pure rotation and translation matrices, $B$ is the coupling one, $B^{T}$ is transpose of the matrix $B$, and

$$
\begin{aligned}
& A=\frac{E I_{x}}{L}\left[\begin{array}{ccc}
k_{11} & 0 & k_{13} \\
0 & k_{22} & 0 \\
k_{31} & 0 & k_{33}
\end{array}\right], \\
& B=\frac{E I_{x}}{L}\left[\begin{array}{ccc}
0 & k_{15} & 0 \\
k_{24} & 0 & k_{26} \\
0 & k_{35} & 0
\end{array}\right], \\
& C=\frac{E I_{x}}{L}\left[\begin{array}{ccc}
k_{44} & 0 & 0 \\
0 & k_{55} & 0 \\
0 & 0 & k_{66}
\end{array}\right] .
\end{aligned}
$$

Then, by (1), we obtain

$$
\begin{aligned}
& \tau=A \phi+B \delta \\
& f=B^{T} \phi+C \delta
\end{aligned}
$$

Since the pure translation matrix $C$ is already a diagonal matrix, the eigenvalues of matrix $C$ are $\left(E I_{x} / L\right) k_{44}$, $\left(E I_{x} / L\right) k_{55}$, and $\left(E I_{x} / L\right) k_{66}$, respectively. Define $K_{T \min }=$ $\min \left\{\left(E I_{x} / L\right) k_{44},\left(E I_{x} / L\right) k_{55},\left(E I_{x} / L\right) k_{66}\right\}$ and $K_{T \max }=$ $\max \left\{\left(E I_{x} / L\right) k_{44},\left(E I_{x} / L\right) k_{55},\left(E I_{x} / L\right) k_{66}\right\}$ as the minimum and maximum translation stiffness for the matrix $C$, respectively. The pure rotation matrix $A$ is symmetric, but not 


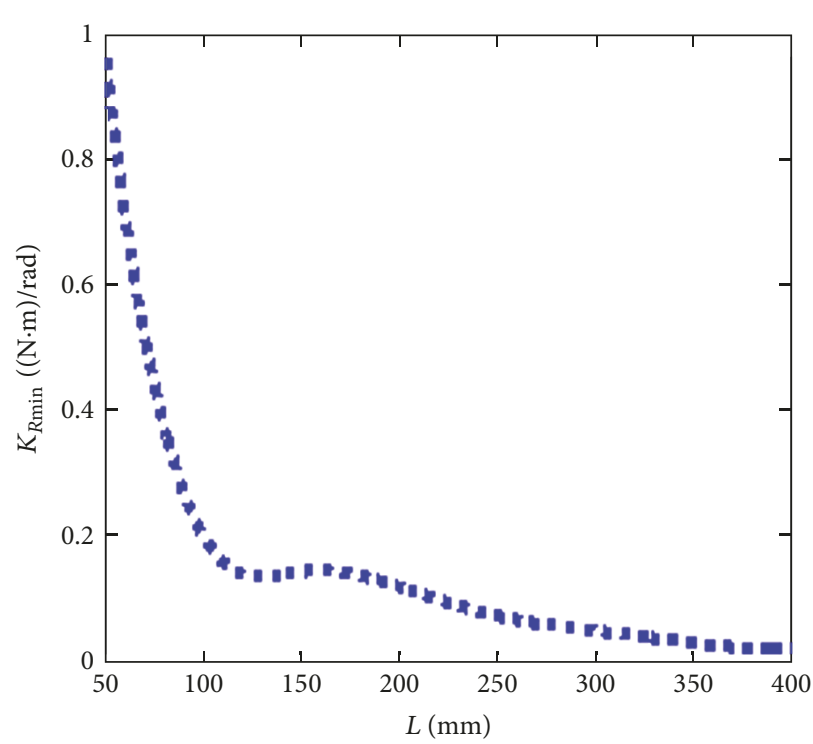

(a)

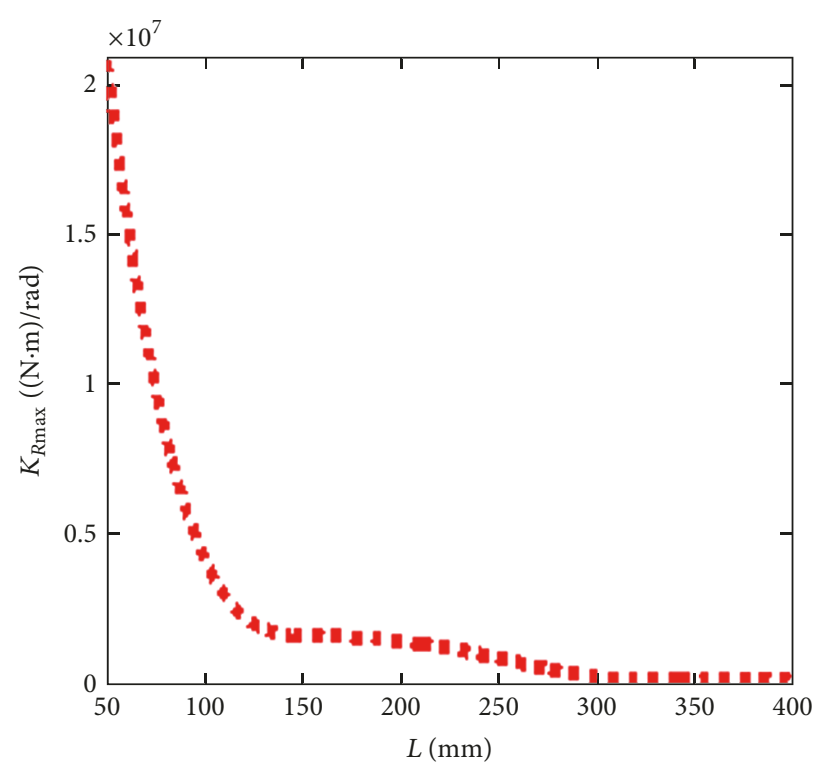

(b)

Figure 4: (a) The influence of parameter $L$ on the minimum rotation stiffness. (b) The influence of parameter $L$ on the maximum rotation stiffness.

a diagonal matrix; thus, there is an orthogonal matrix $Q$, making $Q^{T} A Q$ a diagonal matrix [33]; that is,

$$
Q^{T} A Q=\left[\begin{array}{ccc}
\lambda_{1} & 0 & 0 \\
0 & \lambda_{2} & 0 \\
0 & 0 & \lambda_{3}
\end{array}\right],
$$

where $Q=\left[q_{1}, q_{2}, q_{3}\right] \in \mathrm{SO}(3)$ represents an orthogonal matrix whose columns are just the eigenvectors of matrix $A$ and $Q^{T} A Q=\operatorname{diag}\left[\lambda_{1}, \lambda_{2}, \lambda_{3}\right]$ is a diagonal matrix consisting of the corresponding eigenvalues of matrix $A$. We define $K_{R \min }=\min \left\{\lambda_{1}, \lambda_{2}, \lambda_{3}\right\}$ and $K_{R \max }=\max \left\{\lambda_{1}, \lambda_{2}, \lambda_{3}\right\}$ as the minimum and maximum rotation stiffness for the matrix $A$, respectively.

The matrix $C$ is diagonal matrix, it is easy to obtain the minimum and maximum translation stiffness; therefore, we do not do a lot of analysis here. However, the matrix $A$ is not a diagonal matrix and is coupled, In the following, we analyze the effect of mainly structural parameters $L$ and $r$ on minimum rotation stiffness $K_{R \min }$ and maximum rotation stiffness $K_{R \max }$. The parameters of the NiTi wire are shown in Table 1.

First, the influence of parameter $L$ on the minimum and maximum rotation stiffness is analyzed. For ease of research, assuming that $r$ is a constant and $r=3 \mathrm{~mm}$, the images of minimum and maximum rotation stiffness with the change $L$ are shown in Figure 4.

In Figure 4(a), it can be seen that when $L$ increases, $K_{R \min }$ decreases. When $L \in[50,125] \mathrm{mm}$, the speed of change of $K_{R \min }$ is bigger. When $L \in[125,400] \mathrm{mm}$, the speed of change of $K_{R \min }$ is smaller. In Figure $4(\mathrm{~b})$, when $L$ increases, $K_{R \max }$ decreases. When $L \in[50,150] \mathrm{mm}$, the speed of change of $K_{R \max }$ is bigger. When $L \in[150,400] \mathrm{mm}$,
TABLE 1: The parameters of the NiTi wire.

\begin{tabular}{lc}
\hline Parameter & Value \\
\hline$r_{b}$ & $6 \times 10^{-4} \mathrm{~m}$ \\
$A$ & $1.13 \times 10^{-6} \mathrm{~m}^{2}$ \\
$I_{x}$ & $1.017 \times 10^{-13} \mathrm{~m}^{4}$ \\
$I_{y}$ & $1.017 \times 10^{-13} \mathrm{~m}^{4}$ \\
$J$ & $2.034 \times 10^{-13} \mathrm{~m}^{4}$ \\
$G$ & $2.5 \times 10^{10} \mathrm{~Pa}$ \\
$E$ & $6.5 \times 10^{10} \mathrm{~Pa}$ \\
\hline
\end{tabular}

the speed of change of $K_{R \max }$ is smaller. Considering the minimum and maximum rotation stiffness comprehensively, if $L \in[50,125] \mathrm{mm}$, the influence of $L$ on the minimum and maximum rotation stiffness is bigger. If $L \in[125,400] \mathrm{mm}$, the influence of $L$ on the minimum and maximum rotation stiffness is smaller.

Next, we analyze the influence of parameter $r$ on the minimum and maximum rotation stiffness. Here, it is supposed that $L$ is a constant and $L=200 \mathrm{~mm}$; the minimum and maximum rotation stiffness of the image with the change $r$ are shown in Figure 5.

In Figure 5(a), if $r$ increases, $K_{R \min }$ decreases. When $r \in$ $[1,17] \mathrm{mm}$, the speed of change of $K_{R \min }$ is bigger. When $r \in[17,30] \mathrm{mm}$, the speed of change of $K_{R \min }$ is smaller. In Figure 5(b), when $r$ increases, $K_{R \max }$ decreases. When $r \in[1,10] \mathrm{mm}$, the speed of change of $K_{R \max }$ is bigger. When $r \in[10,30] \mathrm{mm}$, the speed of change of $K_{R \min }$ is smaller. In summary, if $r \in[1,15] \mathrm{mm}$, the influence of $r$ on the minimum and maximum rotation stiffness is bigger. If $r \in[15,30] \mathrm{mm}$, the influence of $r$ on the minimum and maximum rotation stiffness is smaller. 


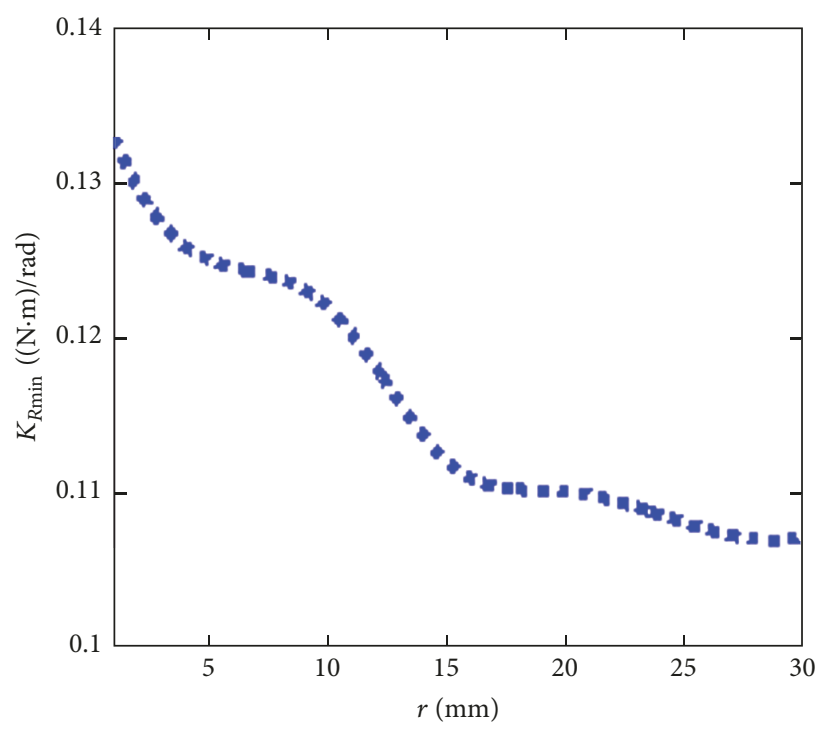

(a)

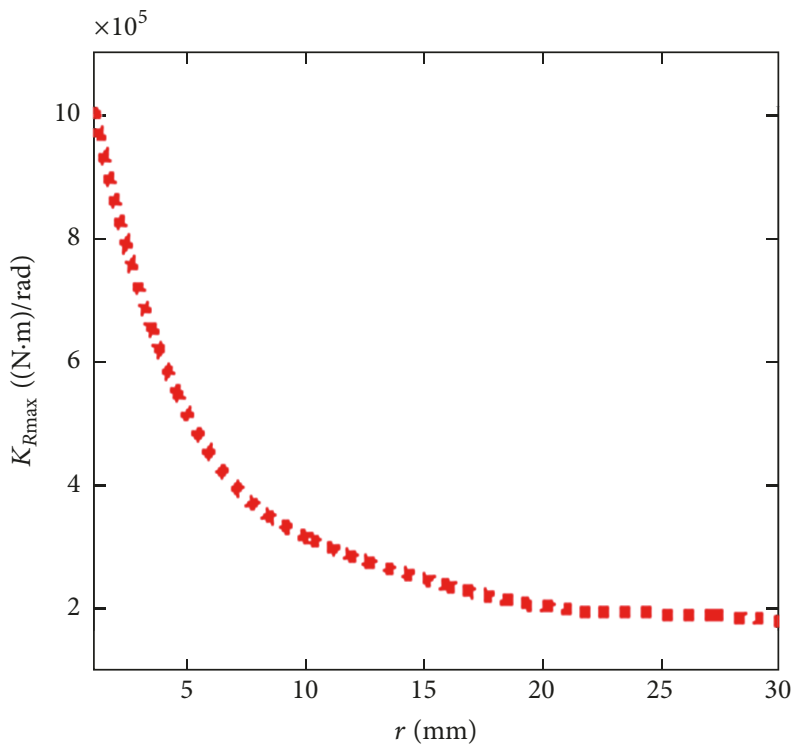

(b)

FIGURE 5: (a) The influence of parameter $r$ on the minimum rotation stiffness. (b) The influence of parameter $r$ on the maximum rotation stiffness.

The block matrices $A$ and $C$ have been analyzed above. Next, the matrix $B$ is researched. In order to facilitate the study, the lemma is first given as follows.

Lemma 1. If a matrix $A$ is an n-order asymmetric matrix, then the matrix A must not be orthogonal to diagonalization.

Proof. Set a matrix $A$ as an $n$-order asymmetric matrix, and suppose there is an $n$-order orthogonal matrix $P$, making

$$
P^{T} A P=\Lambda,
$$

where $\Lambda$ is a diagonal matrix and the diagonal elements are the eigenvalues of the matrix $A$. Transposing (43) yields

$$
\left(P^{T} A P\right)^{T}=\Lambda^{T}
$$

Since

$$
\begin{aligned}
\left(P^{T} A P\right)^{T} & =P^{T} A^{T} P, \\
\Lambda^{T} & =\Lambda,
\end{aligned}
$$

by (43), (49), and (50), we obtain

$$
P^{T} A^{T} P=P^{T} A P .
$$

Multiplying $P$ and its transpose $P^{T}$ of (51) yields

$$
P P^{T} A^{T} P P^{T}=P P^{T} A P P^{T} .
$$

Since the matrix $P$ is an orthogonal matrix, that is, $P P^{T}$ is a unit matrix, then, this yields

$$
A^{T}=A \text {. }
$$

Thus, it is obtained that the matrix $A$ is symmetric matrix. It is inconsistent with the hypothetical matrix $A$ which is an $n$-order asymmetric matrix at the beginning of the proof. Therefore, the matrix $A$ cannot be orthogonal to diagonalization.

The matrix $B$ reflects the relationship between $\tau$ and $\delta$, and the units of each element in the matrix $B$ are consistent. However, the matrix $B$ is not a symmetric matrix in (40), by Lemma 1, obtaining that matrix $B$ cannot be orthogonal to diagonalization. Therefore, the matrix $B$ has no minimum eigenvalue and maximum eigenvalue. Here, we cannot define the minimum stiffness and maximum stiffness of matrix $B$. The transpose matrix $B^{T}$ also cannot define the minimum stiffness and maximum stiffness.

4.3. Numerical Analysis of Stiffness Elements. In this section, in order to understand the stiffness elements of continuum robot more intuitively, the stiffness elements of continuum robot are analyzed by the numerical example. Here, we select the structural parameters of continuum robot such as $L=$ $210 \mathrm{~mm}, r=5 \mathrm{~mm}$. The parameters of NiTi wire are shown in Table 1.

The results are tabulated in Table 2, where the unit for force component is Newton, the unit of moment is $\mathrm{N} \cdot \mathrm{m}$, and the units for translational and rotational displacements are meter and radian, respectively. By analyzing the numerical results, the following conclusions can be obtained.

(1) The rotational stiffness elements $\tau_{x} / \phi_{x}$ and $\tau_{y} / \phi_{y}$ are approximately equal. It is indicated that the same moment is, respectively, applied around the axes $x$ and $y$ at the end of continuum robot; then the rotational angles of the end are the approximately equal. Moreover, $\tau_{x} / \phi_{x}$ is about 94.88 times larger than $\tau_{y} / \delta_{z}$, and $\tau_{y} / \phi_{y}$ is about 94.47 times larger than $\tau_{y} / \delta_{z}$. It is shown that the rotation around the axes $x$ and $y$ is harder than that around the axis $z$. 
TABLE 2: Numerical results of stiffness elements.

\begin{tabular}{lc}
\hline Stiffness & Value \\
element & 9.26 \\
\hline$\tau_{x} / \phi_{x}$ & $-1.86 \times 10^{-3}$ \\
$\tau_{x} / \phi_{z}$ & 3.6 \\
$\tau_{x} / \delta_{y}$ & 9.22 \\
$\tau_{y} / \phi_{y}$ & -3.6 \\
$\tau_{y} / \delta_{x}$ & $2.48 \times 10^{3}$ \\
$\tau_{y} / \delta_{z}$ & $-1.86 \times 10^{-3}$ \\
$\tau_{z} / \phi_{x}$ & $9.76 \times 10^{-2}$ \\
$\tau_{z} / \phi_{z}$ & $-1.77 \times 10^{-2}$ \\
$\tau_{z} / \delta_{y}$ & -3.6 \\
$f_{x} / \phi_{y}$ & $3.43 \times 10$ \\
$f_{x} / \delta_{x}$ & 3.6 \\
$f_{y} / \phi_{x}$ & $-1.77 \times 10^{-2}$ \\
$f_{y} / \phi_{z}$ & $3.43 \times 10$ \\
$f_{y} / \delta_{y}$ & $2.48 \times 10^{3}$ \\
$f_{z} / \phi_{y}$ & $1.05 \times 10^{6}$ \\
$f_{z} / \delta_{z}$ &
\end{tabular}

Major stiffness elements. Elements not listed are zeros; Units: $\tau_{x, y, z}(\mathrm{~N} \cdot \mathrm{m})$, $f_{x, y, z}(\mathrm{~N}), \phi_{x, y, z}(\mathrm{rad}), \delta_{x, y, z}(\mathrm{~m})$.

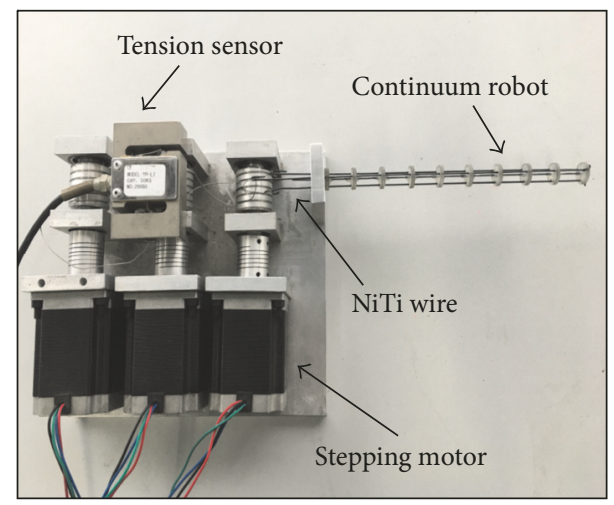

FIgURE 6: A single section NiTi wire continuum robot.

(2) The translational stiffness elements $f_{x} / \delta_{x}$ and $f_{y} / \delta_{y}$ are equal. It is shown that the same force is, respectively, applied along the axes $x$ and $y$ at the end of continuum robot; then the translational distances of the end are equal. Meanwhile, the translational stiffness element $f_{z} / \delta_{z}=1.05 \times$ $10^{6}(\mathrm{~N} / \mathrm{m})$ is significantly larger than translational stiffness element $f_{x} / \delta_{x}=f_{y} / \delta_{y}=3.43 \times 10(\mathrm{~N} / \mathrm{m})$. It is indicated that the translation along the axis $z$ is harder than translation along the axes $x$ and $y$, respectively. It all fits our intuition.

\section{Experiments}

The proposed stiffness model is validated by a single section NiTi wire driving continuum robot, as shown in Figure 6. The structural parameters of continuum robot are $L=210 \mathrm{~mm}$, $r=5 \mathrm{~mm}$. The parameters of NiTi wire are shown in Table 1 . The disks are made of 3D printed machine (EDEN-260V)

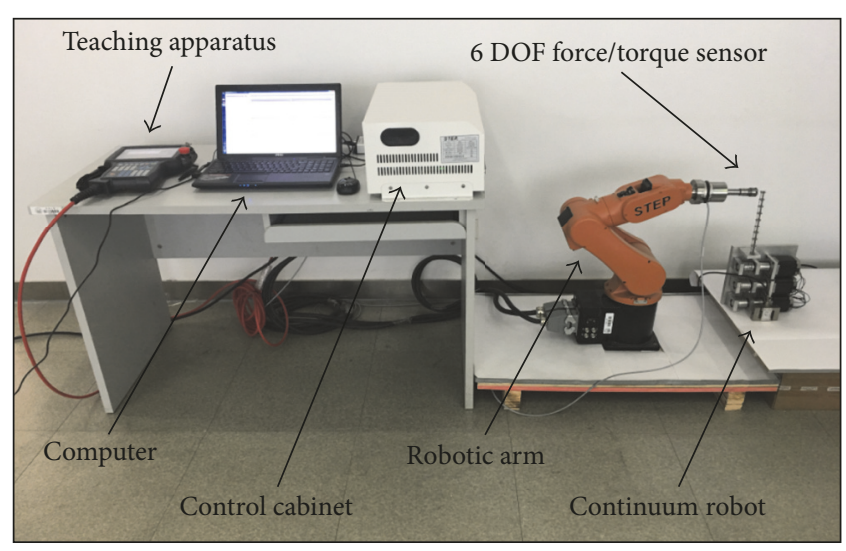

FIgURE 7: Experimental setup for stiffness experiments.

with hard material (VeroClear RGD810), and the disks are very hard. The secondary backbones are connected with three stepping motors, which produce the driving force to bend the continuum robot. The tension sensor is used to record the driving force which is produced by motors.

The experimental setup is shown as Figure 7, which consists of a continuum robot, a control cabinet, a teaching apparatus, a robotic arm, a computer, and a force/torque sensor. The robotic arm (STEP-SD500) is used to provide an external load at the end of continuum robot, and the $6 \mathrm{DOF}$ force/torque commercial sensor (OPTOFORCE-HEX-70$\mathrm{XE}-450$ ) is mounted at the end of robotic arm to record the external load force.

Here, we facilitate the experimental operations by verifying the compliance matrix to indicate that the stiffness matrix is correct. Meanwhile, we pay more attention to the relations between forces and deformations at the end of continuum robot along the three translational directions. Therefore, we operate the experiment to verify the relations between forces and deformations in three translational directions. It is assumed that the external forces applied at the end of continuum robot are twists $\xi_{1}, \xi_{2}$, and $\xi_{3}$, where $\xi_{1}=\left(0,0,0, f_{x}, 0,0\right)^{T}, \xi_{2}=\left(0,0,0,0, f_{y}, 0\right)^{T}$, and $\xi_{3}=$ $\left(0,0,0,0,0, f_{z}\right)^{T}$. Substituting twists $\xi_{1}, \xi_{2}$, and $\xi_{3}$ into (20), respectively, we obtain external load force and translational distance that meet the following relationship:

$$
\begin{aligned}
& \delta_{x}=2.785 f_{x} \\
& \delta_{y}=2.624 f_{y} \\
& \delta_{z}=9.5 \times 10^{-4} f_{z},
\end{aligned}
$$

where $f_{x}(\mathrm{~N}), f_{y}(\mathrm{~N}), f_{z}(\mathrm{~N}), \delta_{x}(\mathrm{~cm}), \delta_{y}(\mathrm{~cm})$, and $\delta_{z}(\mathrm{~mm})$.

Next, through the experiments we verify that (49), (50), and (51) are correct. The experiments were done at low moving speed to reduce the effect of vibration. First, the experiments along the axis $x$ are shown in Figure 8. The force and translational distance at the end of continuum robot is recorded by $6 \mathrm{DOF}$ force/torque commercial sensor and the operation of robotic arm, respectively. 


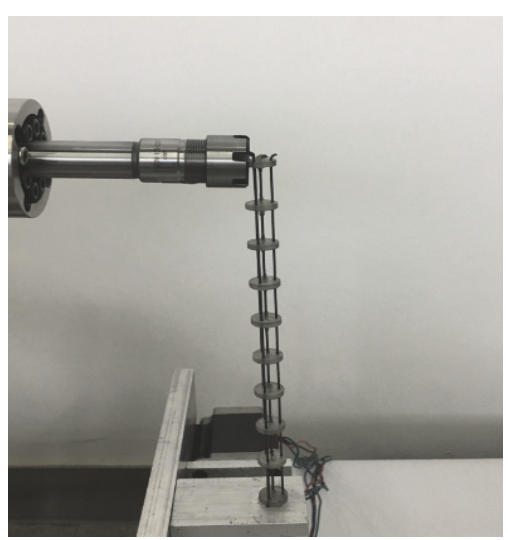

(a)

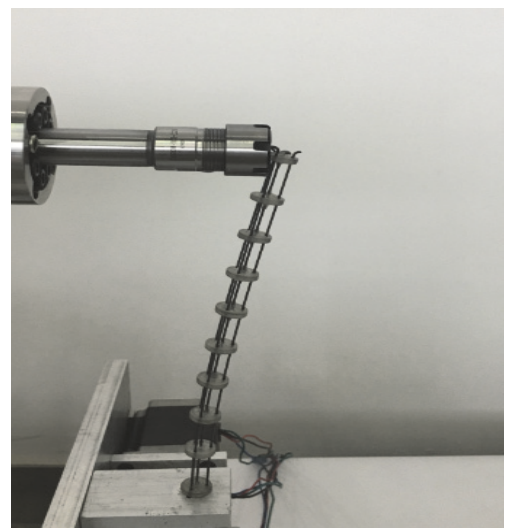

(d)

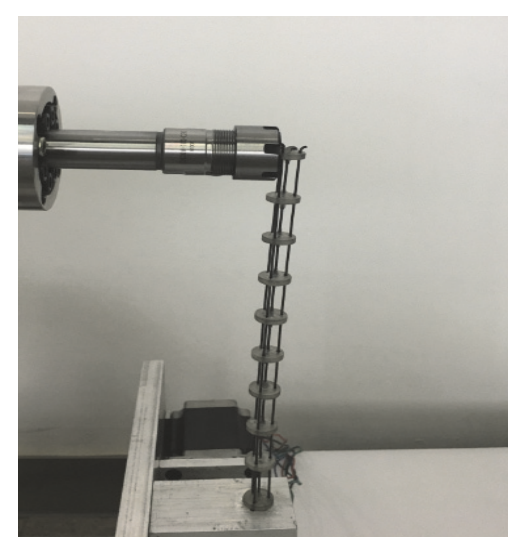

(b)

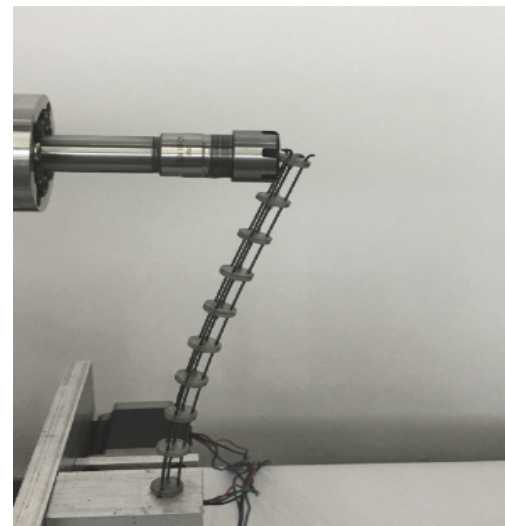

(e)

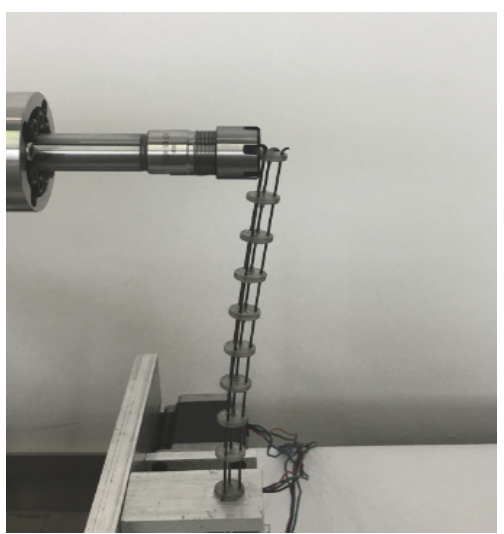

(c)

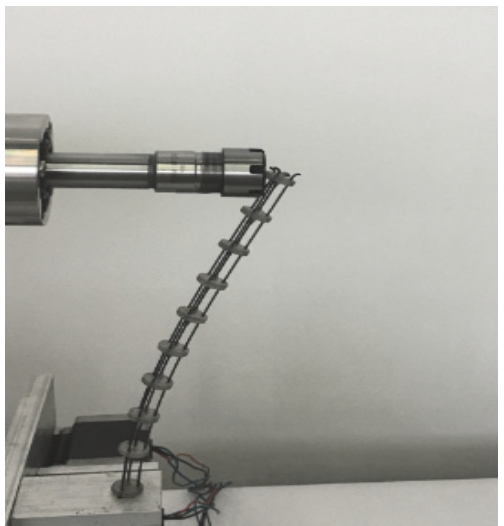

(f)

FIGURE 8: The experiments along the axis $x$.

The experimental values and the functional image of (49) are shown in Figure 9. The experimental values are distributed around the theoretical image, and the errors between the theoretical values and the experimental values are no more than $4 \%$, thus, indicating that (49) is correct.

Then, the experiments along the axis $y$ are shown in Figure 10.

The experimental values and the functional image of (50) are shown in Figure 11. The errors between the theoretical values and the experimental values are no more than $5 \%$, therefore, demonstrating that (50) is correct.

Finally, the experiments along the axis $z$ are shown in Figure 12.

The experimental values and the functional image of (51) are shown in Figure 13. The errors between the theoretical values and the experimental values are no more than $7 \%$, thence, showing that (51) is correct.

Through the above experiments, we verify that the translational stiffness of continuum robot along the axes $x, y$, and $z$, respectively, is correct. By comparing the translational experimental values along the axes $x$ and $y$, respectively, if the external force $f_{x}=f_{y}$, the translational distances $\delta_{x}$ and $\delta_{y}$ are approximately equal. By comparing the translational experimental values along the axes $x$ and $z$, respectively, if the external force $f_{x}=f_{z}$, the translational distance $\delta_{x}$ is much larger than translational distance $\delta_{z}$; it also fits our intuitive perception.

\section{Discussions}

In this paper, we mainly establish the stiffness model of a single section multibackbone continuum robot, with the initial configuration of continuum robot being a straight line and $L_{i}=L(i=1,2,3)$. If the continuum robot is multisection and the initial configuration of continuum robot is a straight line, then the stiffness model of a multisection continuum robot can be studied by each lower section as a payload of its upper sections. In addition, the stiffness model of continuum robot is based on the initial configuration which is a straight line. If the initial configuration of continuum robot is a bending shape, then the length of the four NiTi wires of continuum robot is not equal. Thus, the stiffness model in (18) is not suitable for analyzing the load model of continuum robot. Simultaneously, it should be pointed out that the deformations of all backbones should be small; otherwise, the elastic properties of the backbone are damaged; then the stiffness model in (18) is also not suitable for analyzing the load model of continuum robot.

In our model, the $6 \times 6$ stiffness matrix in (18) indicates relationship between force/moment and motion/rotation at 


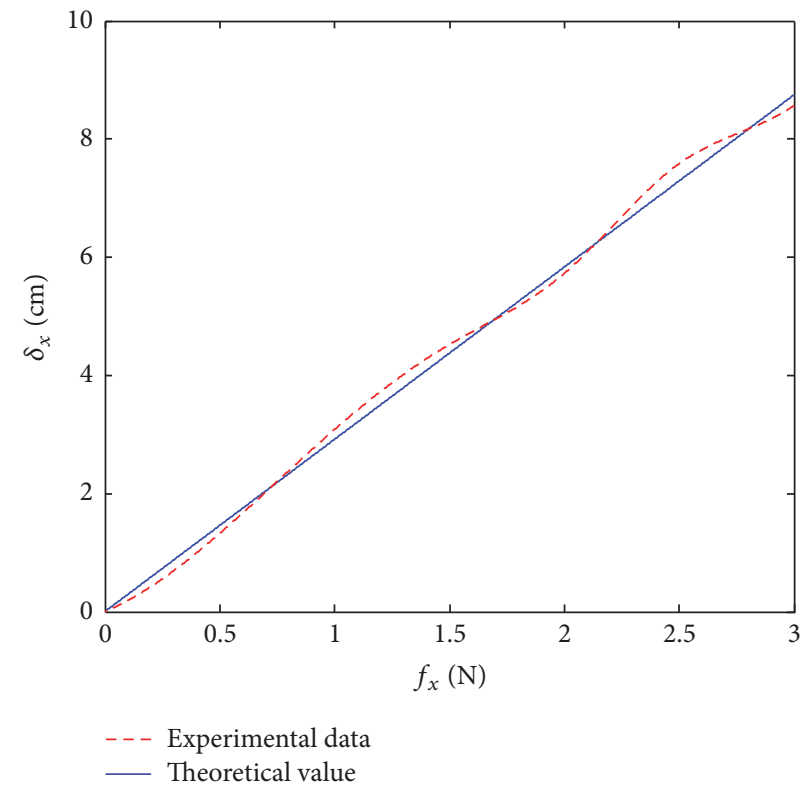

FIGURE 9: Theoretical and experimental values along the axis $x$.

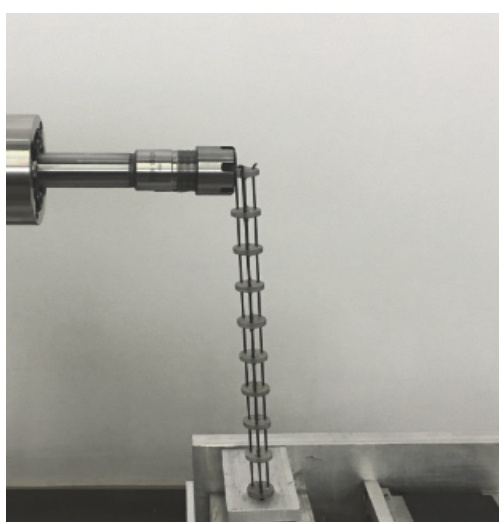

(a)

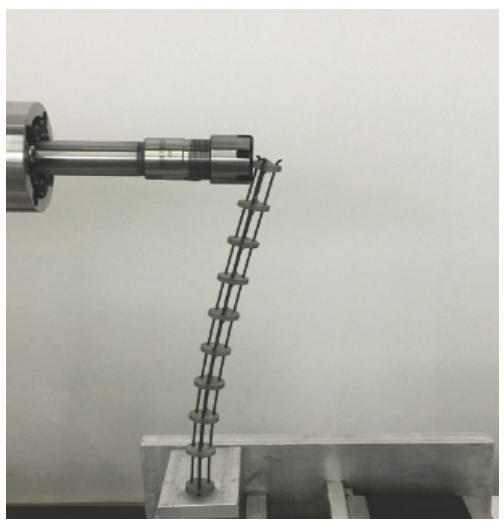

(d)

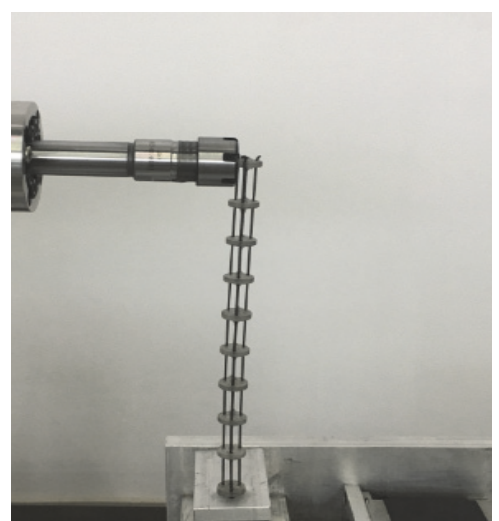

(b)

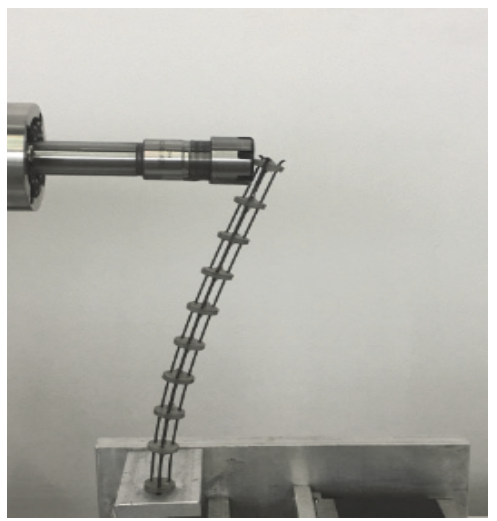

(e)

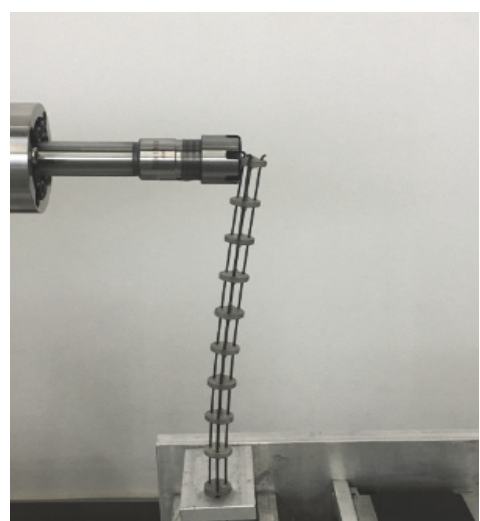

(c)

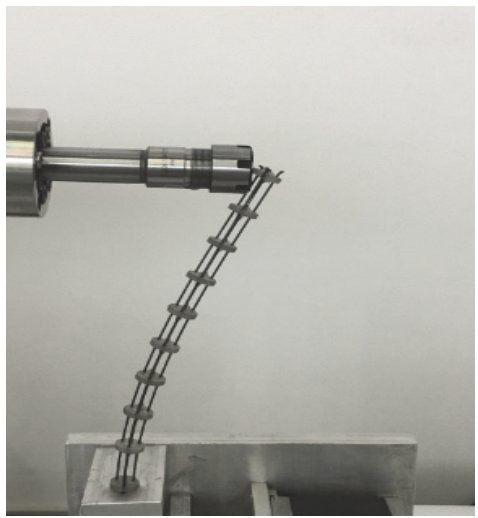

(f)

FIgURE 10: The experiments along the axis $y$. 


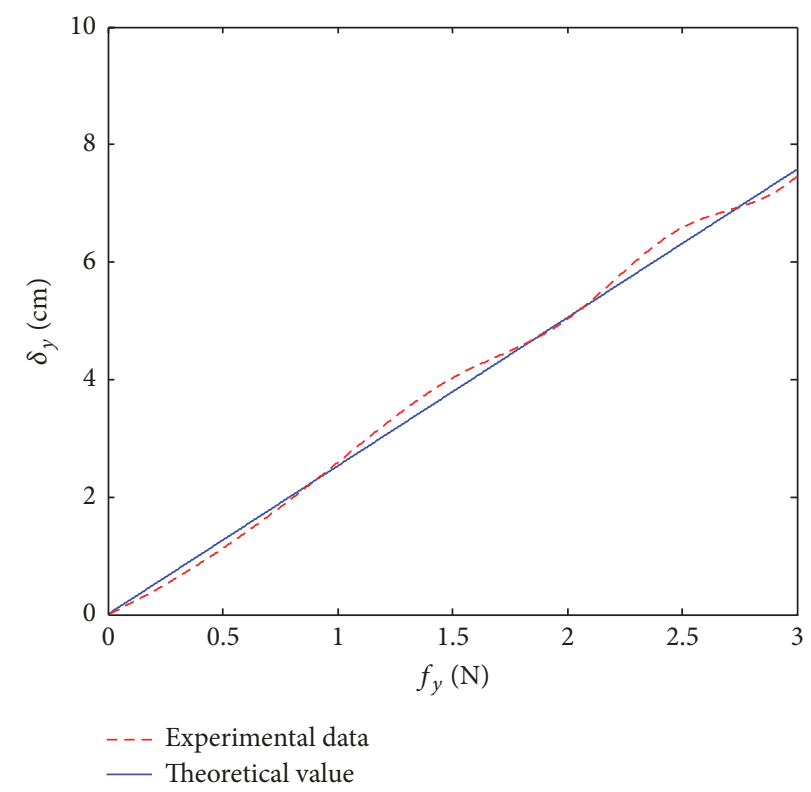

FIGURE 11: Theoretical and experimental values along the axis $y$.

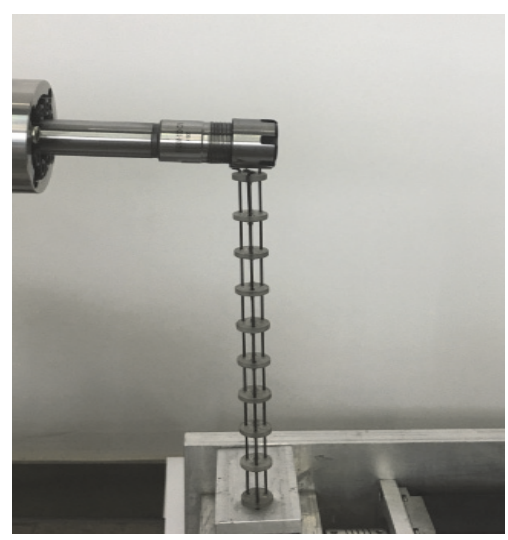

(a)

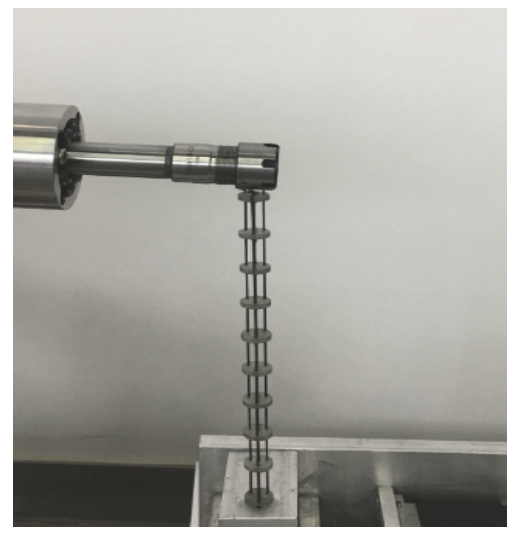

(d)

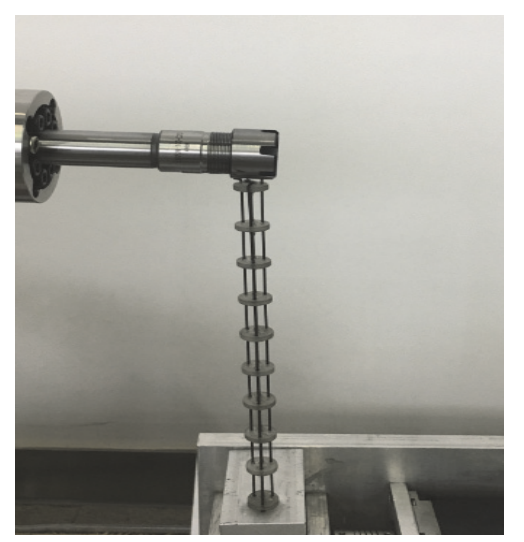

(b)

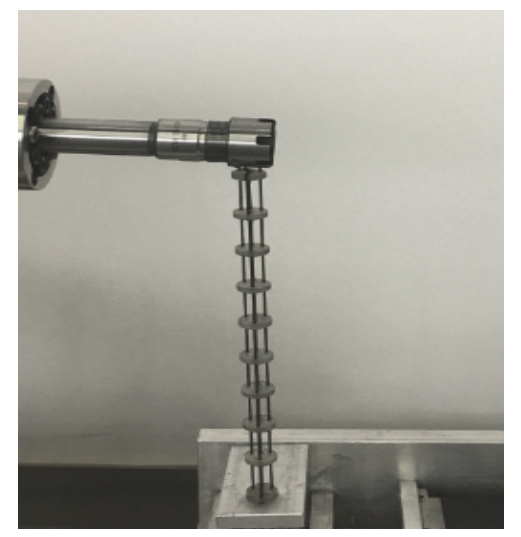

(e)

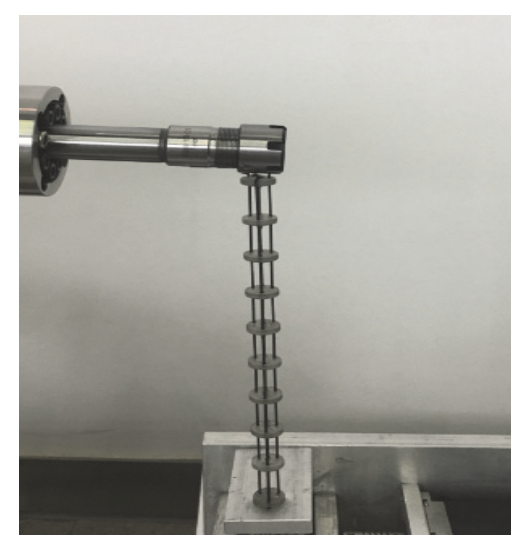

(c)

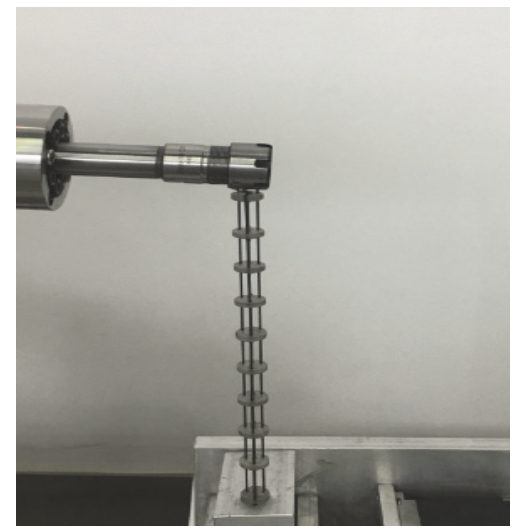

(f)

FIgURE 12: The experiments along the axis $z$. 


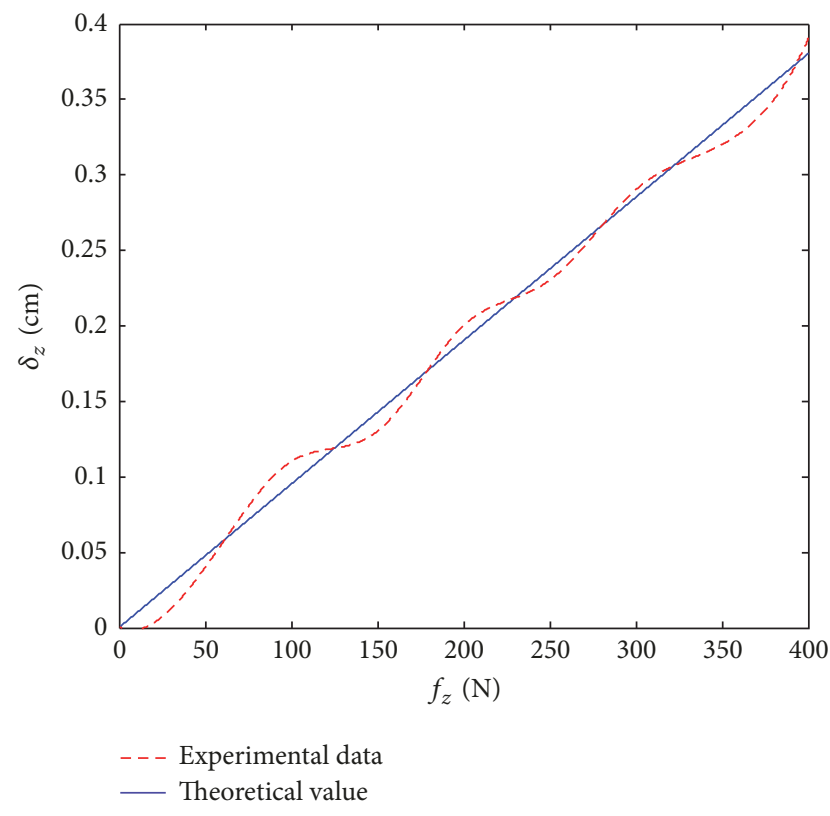

FIGURE 13: Theoretical and experimental values along the axis $z$.

the end of continuum robot. If the end of continuum robot is subjected to force $f_{x}$ along the forward direction of the axis $x$, the shape of continuum robot at this time is defined as $A$. Next, on the basis of shape $A$, the force $f_{y}$ is applied to the end of continuum robot along the forward direction of the axis $y$; then the final bending shape of continuum robot is defined as $B$. The shape $B$ is equivalent to the resultant force of forces $f_{x}$ and $f_{y}$, which is applied to the end of continuum robot; the end position of continuum robot with the shape $B$ can be obtained by substituting $f_{x}$ and $f_{y}$ into the stiffness matrix of (18). Furthermore, on the basis of the shape $A$, if the force $f_{z}$ is applied to the end of continuum robot along the opposite direction of the axis $z$, then the final bending shape of continuum robot is defined as $C$. However, the end position of continuum robot with the shape $C$ cannot be obtained by substituting $f_{x}$ and $f_{z}$ into the stiffness matrix of (18). Therefore the stiffness element $k_{p 66}=f_{z} / \delta_{z}$ is only applicable to the case in which the continuum robot does not have any deformations. Through the same analytical method, the stiffness element $k_{p 33}=\tau_{z} / \phi_{z}$ is only applicable to the case in which the continuum robot does not have any deformations. However, the other main stiffness elements $k_{p 11}, k_{p 22}, k_{p 44}$, and $k_{p 55}$ are applicable to the case in which the continuum robot is deformed and nondeformed.

\section{Conclusions}

In this paper, the main contribution is to propose a simplified method to establish the stiffness model of a single section multibackbone continuum robot, so as to obtain the relationship between force and deformation at the end of continuum. First, the stiffness model of continuum robot is established by means of the screw theory and Euler-Bernoulli beam. Second, the stiffness elements of the stiffness model are analyzed, obtaining the influence of the structural parameters of continuum robot on the stiffness elements. Third, the minimum and maximum rotation/translation stiffness are defined to analyze the effect of the structural parameters of continuum robot on the overall rotation and translation stiffness. In addition, the stiffness elements of continuum robot are analyzed by the numerical example. Finally, the stiffness model is verified by experiments; simultaneously, the experimental results indicate that the proposed stiffness model of continuum robot is correct. The research of this paper fully indicates the relationship between the force and deformation at the end of continuum robot and, thus, provides an effective theoretical basis for the real-time control of continuum robot.

\section{Conflicts of Interest}

The authors declare that they have no conflicts of interest regarding the publication of this paper.

\section{Acknowledgments}

The work was supported by National Natural Science Foundation of China (Grants nos. 51275360 and 51538009) and Excellent Technology Leadership Program of Shanghai (Grant no. 15XD1525000).

\section{References}

[1] M. M. Tonapi, I. S. Godage, A. M. Vijaykumar, and I. D. Walker, "A novel continuum robotic cable aimed at applications in space," Advanced Robotics, vol. 29, no. 13, pp. 861-875, 2015.

[2] I. S. Godage, G. A. Medrano-Cerda, D. T. Branson, E. Guglielmino, and D. G. Caldwell, "Modal kinematics for multisection continuum arms," Bioinspiration \& Biomimetics, vol. 10, no. 3, Article ID 035002, 2015.

[3] M. Moradi Dalvand, S. Nahavandi, and R. D. Howe, "Fast vision-based catheter 3D reconstruction," Physics in Medicine and Biology, vol. 61, no. 14, article no. 5128, pp. 5128-5148, 2016.

[4] W. S. Rone and P. Ben-Tzvi, "Mechanics modeling of multisegment Rod-Driven continuum robots," Journal of Mechanisms and Robotics, vol. 6, no. 4, Article ID 041006, 2014.

[5] S. Song, Z. Li, H. Yu, and H. Ren, "Shape reconstruction for wire-driven flexible robots based on Bézier curve and electromagnetic positioning," Mechatronics, vol. 29, pp. 28-35, 2015.

[6] M. Ivanescu, D. Popescu, and N. Popescu, "A decoupled sliding mode control for a continuum arm," Advanced Robotics, vol. 29, no. 13, pp. 831-845, 2015.

[7] H. B. Gilbert, J. Neimat, and R. J. Webster, "Concentric Tube Robots as Steerable Needles: Achieving Follow-the-Leader Deployment," IEEE Transactions on Robotics, vol. 31, no. 2, pp. 246-258, 2015.

[8] Z. Du, W. Yang, and W. Dong, "Kinematics modeling and performance optimization of a kinematic-mechanics coupled continuum manipulator," Mechatronics, vol. 31, pp. 196-204, 2015.

[9] Y. Tian, M. Luan, X. Gao, W. Wang, and L. Li, "Kinematic analysis of continuum robot consisted of driven flexible rods," Mathematical Problems in Engineering, vol. 2016, Article ID 6984194, 2016. 
[10] C. Bergeles, A. H. Gosline, N. V. Vasilyev, P. J. Codd, P. J. Del Nido, and P. E. Dupont, "Concentric tube robot design and optimization based on task and anatomical constraints," IEEE Transactions on Robotics, vol. 31, no. 1, pp. 67-84, 2015.

[11] Z. Li, H. Ren, P. W. Y. Chiu, R. Du, and H. Yu, "A novel constrained wire-driven flexible mechanism and its kinematic analysis," Mechanism and Machine Theory, vol. 95, pp. 59-75, 2016.

[12] B. He, Z. Wang, Q. Li, H. Xie, and R. Shen, "An analytic method for the kinematics and dynamics of a multiple-backbone continuum robot," International Journal of Advanced Robotic Systems, vol. 10, article no. 84, 2013.

[13] D. Trivedi, C. D. Rahn, W. M. Kier, and I. D. Walker, "Soft robotics: biological inspiration, state of the art, and future research," Applied Bionics and Biomechanics, vol. 5, no. 3, pp. 99-117, 2008.

[14] R. J. Webster III and B. A. Jones, "Design and kinematic modeling of constant curvature continuum robots: a review," International Journal of Robotics Research, vol. 29, no. 13, pp. 1661-1683, 2010.

[15] J. M. Selig and X. Ding, "A screw theory of timoshenko beams," Journal of Applied Mechanics, vol. 76, no. 3, pp. 1-7, 2009.

[16] J. S. Dai, "Finite Displacement Screw Operators With Embedded Chasles' Motion," Journal of Mechanisms and Robotics, vol. 4, no. 4, p. 041002, 2012.

[17] X. Pei, J. Yu, G. Zong, S. Bi, and H. Su, "The modeling of cartwheel flexural hinges," Mechanism and Machine Theory, vol. 44, no. 10, pp. 1900-1909, 2009.

[18] X. Ding and J. S. Dai, "Compliance analysis of mechanisms with spatial continuous compliance in the context of screw theory and Lie groups," Proceedings of the Institution of Mechanical Engineers, Part C: Journal of Mechanical Engineering Science, vol. 224, no. 11, pp. 2493-2504, 2010.

[19] S. Awtar and S. Sen, "A generalized constraint model for two-dimensional beam flexures: Nonlinear load-displacement formulation," Journal of Mechanical Design, vol. 132, no. 8, pp. 0810081-08100811, 2010.

[20] G. Krishnan, C. Kim, and S. Kota, "An intrinsic geometric framework for the building block synthesis of single point compliant mechanisms," Journal of Mechanisms and Robotics, vol. 3, no. 1, Article ID 011001, 2010.

[21] I. Tunay, "Spatial continuum models of rods undergoing large deformation and inflation," IEEE Transactions on Robotics, vol. 29, no. 2, pp. 297-307, 2013.

[22] G. Gao, H. Wang, Q. Xia, M. Song, and H. Ren, "Study on the load capacity of a single-section continuum manipulator," Mechanism and Machine Theory, vol. 104, pp. 313-326, 2016.

[23] P. Qi, C. Qiu, H. Liu, J. S. Dai, L. D. Seneviratne, and K. Althoefer, "A Novel Continuum Manipulator Design Using Serially Connected Double-Layer Planar Springs," IEEE/ASME Transactions on Mechatronics, vol. 21, no. 3, pp. 1281-1292, 2016.

[24] I. A. Gravagne, C. D. Rahn, and I. D. Walker, "Large deflection dynamics and control for planar continuum robots," IEEE/ASME Transactions on Mechatronics, vol. 8, no. 2, pp. 299307, 2003.

[25] D. Trivedi, A. Lotfi, and C. D. Rahn, "Geometrically exact dynamic models for soft robotic manipulators," in Proceedings of the 2007 IEEE/RSJ International Conference on Intelligent Robots and Systems, IROS 2007, pp. 1497-1502, usa, November 2007.

[26] D. B. Camarillo, C. F. Milne, C. R. Carlson, M. R. Zinn, and J. K. Salisbury, "Mechanics modeling of tendon-driven continuum manipulators," IEEE Transactions on Robotics, vol. 24, no. 6, pp. 1262-1273, 2008.

[27] J. Fras, J. Czarnowski, M. Macias, and J. Glowka, "Static modeling of multisection soft continuum manipulator for stiffflop project," Advances in Intelligent Systems and Computing, vol. 267, pp. 365-375, 2014.

[28] J. Till and D. C. Rucker, "Elastic rod dynamics: Validation of a real-time implicit approach," in Proceedings of the 2017 IEEE/RSJ International Conference on Intelligent Robots and Systems (IROS), pp. 3013-3019, Vancouver, BC, September 2017.

[29] S. M. Hadi Sadati, S. E. Naghibi, I. D. Walker, K. Althoefer, and T. Nanayakkara, "Control Space Reduction and Real-Time Accurate Modeling of Continuum Manipulators Using Ritz and Ritz-Galerkin Methods," IEEE Robotics and Automation Letters, vol. 3, no. 1, pp. 328-335, 2018.

[30] T. Huang, X. Zhao, and D. J. Whitehouse, "Stiffness estimation of a tripod-based parallel kinematic machine," IEEE Transactions on Robotics and Automation, vol. 18, no. 1, pp. 50-58, 2002.

[31] M. Henke and G. Gerlach, "A multi-layered variable stiffness device based on smart form closure actuators," Journal of Intelligent Material Systems and Structures, vol. 27, no. 3, pp. 375383, 2016.

[32] M. Ceccarelli and G. Carbone, "A stiffness analysis for CaPaMan (Cassino Parallel Manipulator)," Mechanism and Machine Theory, vol. 37, no. 5, pp. 427-439, 2002.

[33] G. Chen, H. Wang, Z. Lin, and X. Lai, "The principal axes decomposition of spatial stiffness matrices," IEEE Transactions on Robotics, vol. 31, no. 1, pp. 191-207, 2015. 


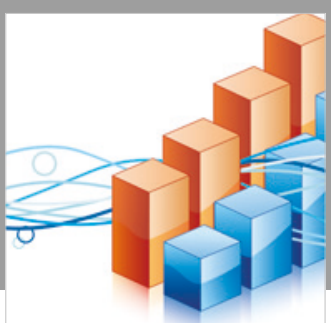

Advances in

Operations Research

\section{-n-m}
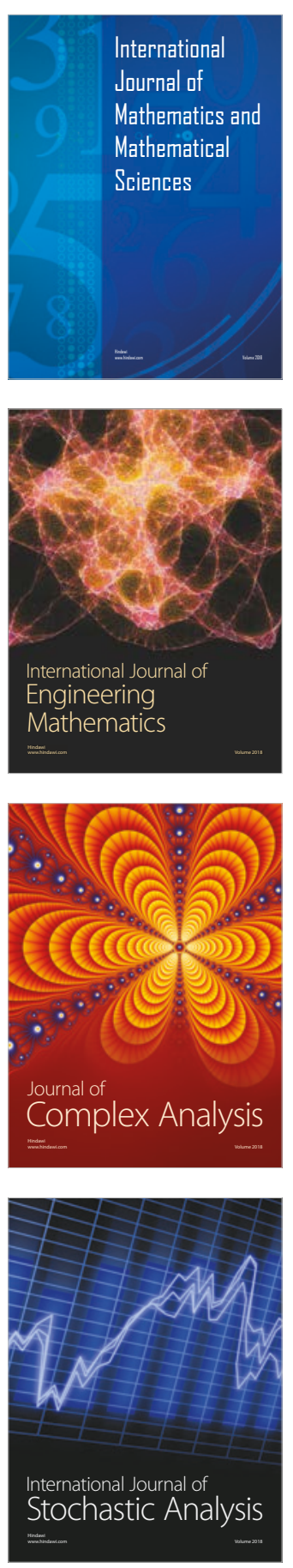
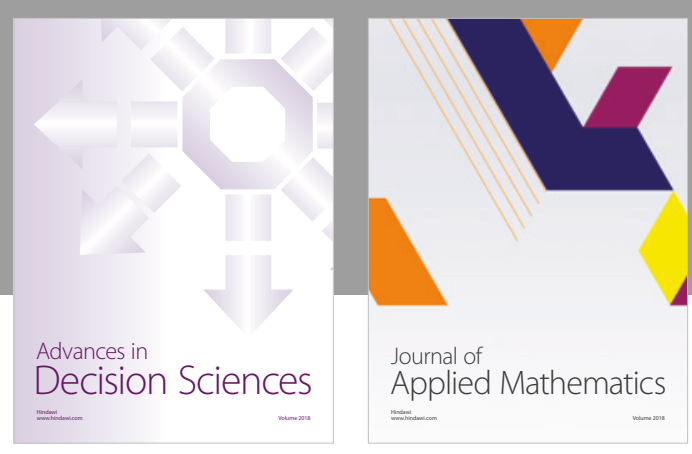

Journal of

Applied Mathematics
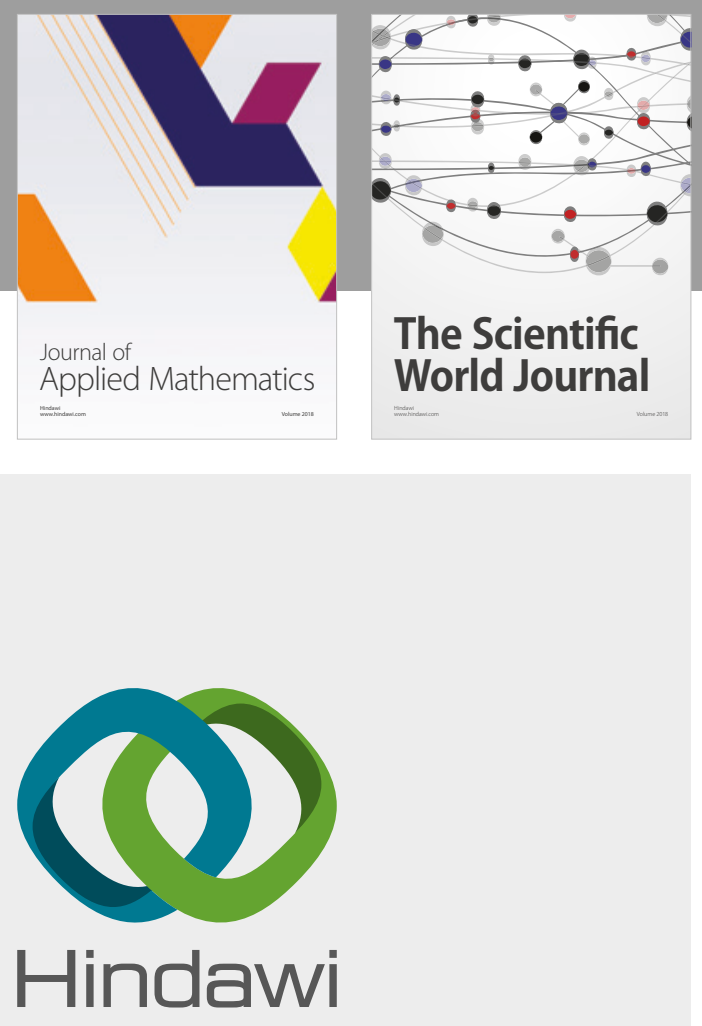

Submit your manuscripts at

www.hindawi.com

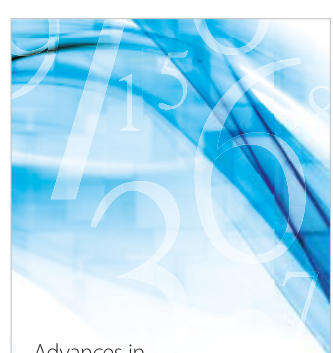

Advances in
Numerical Analysis
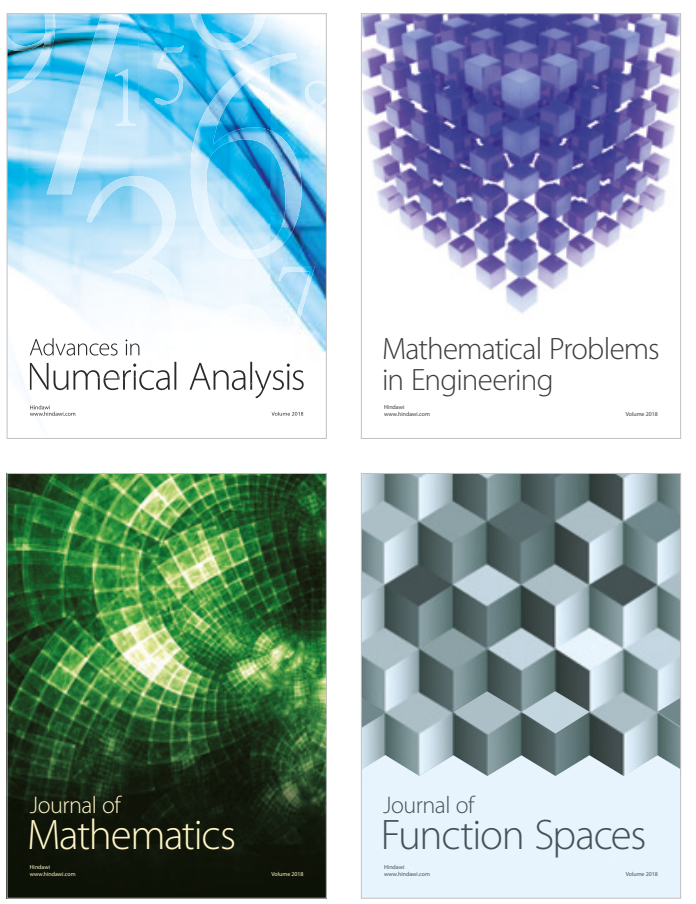

Mathematical Problems in Engineering

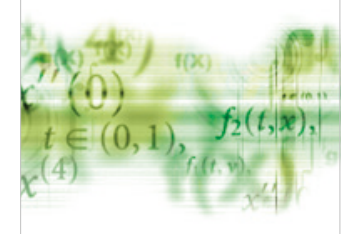

International Journal of

Differential Equations

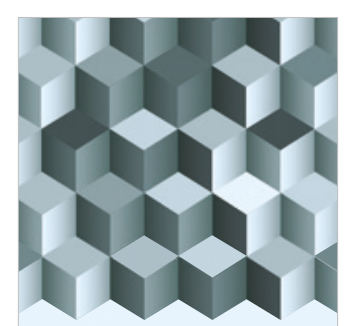

Journal of

Function Spaces

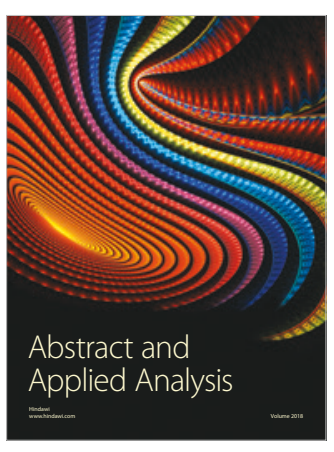

The Scientific

World Journal

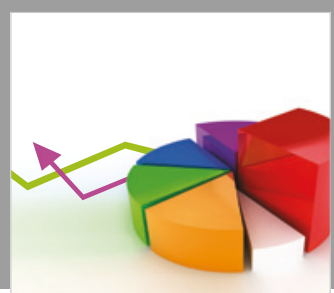

Journal of

Probability and Statistics
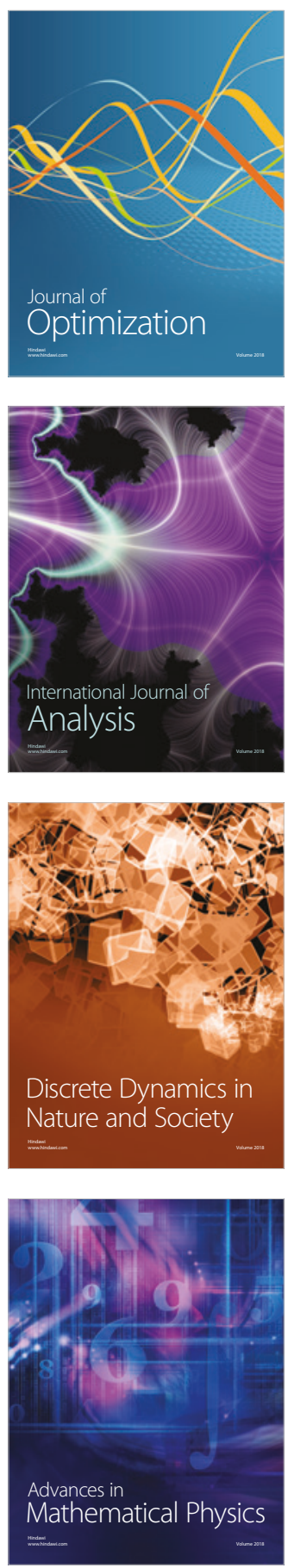